\title{
ASYMPTOTIC ANALYSIS OF AN ADVECTION-DOMINATED CHEMOTAXIS MODEL IN MULTIPLE SPATIAL DIMENSIONS*
}

\author{
MARTIN BURGER ${ }^{\dagger}$, YASMIN DOLAK-STRUSS $^{\ddagger}$, AND CHRISTIAN SCHMEISER $^{\S}$
}

Abstract. This paper is devoted to a study of the asymptotic behavior of solutions of a chemotaxis model with logistic terms in multiple spatial dimensions. Of particular interest is the practically relevant case of small diffusivity, where (as in the one-dimensional case) the cell densities form plateau-like solutions for large time.

The major difference from the one-dimensional case is the motion of these plateau-like solutions. with respect to the plateau boundaries separating zero density regions from maximum density regions. This interface motion appears on a non-logarithmic time scale and can be interpreted as a surface diffusion law. The biological interpretation of the surface diffusion is that a packed region of cells can change its shape mainly if cells diffuse along its boundary.

The theoretical results on the asymptotic behavior are supplemented by several numerical simulations on two- and three-dimensional domains.

Key words. chemotaxis models, asymptotic behavior, interface motion, surface diffusion

AMS subject classifications. 35Q80, 35R35, 92C17

\section{Introduction}

In this paper, we will be concerned with the long-time behavior of the nonlinear chemotaxis model

$$
\begin{aligned}
\partial_{t} \varrho+\nabla_{x} \cdot\left(\varrho(1-\varrho) \nabla_{x} S-\varepsilon \nabla_{x} \varrho\right) & =0, \\
\Delta S & =S-\varrho .
\end{aligned}
$$

In particular, we consider system (1.1), (1.2) for $x \in \Omega \subset \mathbb{R}^{d}, d>1$, and $t>0$, subject to the initial condition

$$
\varrho(x, 0)=\varrho_{I}(x)
$$

and homogeneous Neumann boundary conditions

$$
\partial_{\nu} \varrho=0 \quad \text { and } \quad \partial_{\nu} S=0 \quad \text { on } \partial \Omega,
$$

where $\nu$ is the unit outer normal on $\partial \Omega$, which we assume to be smooth. Equation (1.1) describes the evolution of the cell density $\varrho(x, t)$ under the influence of the chemoattractant $S(x, t)$, when cell motion is governed by chemotaxis, i.e., the directed migration of cells along chemical gradients, and diffusion, described by Fick's law. In the following, we will always assume that the effect of the cells' random movement is small compared to their chemotactic orientation, and choose $0<\varepsilon \ll 1$. In Equation (1.2), the chemoattractant concentration $S$ is subject to diffusion as well as (linear) production by the cells and degradation. In contrast to the so-called classical model

\footnotetext{
* Received: February 28, 2007; accepted (in revised version): October 26, 2007. Communicated by Paul Milewski.

${ }^{\dagger}$ Institut für Numerische und Angewandte Mathematik, Fachbereich Mathematik und Informatik, Westfälische Wilhelms Universität (WWU) Münster, Einsteinstra e 62, D 48149 Münster, Germany (martin.burger@uni-muenster.de).

${ }^{\ddagger}$ Institute for Mathematics, University of Vienna, Nordbergstrasse 15, 1090 Vienna, Austria (yasmin.dolak-struss@univie.ac.at).

$\S$ Institute for Mathematics, University of Vienna, Nordbergstrasse 15, 1090 Vienna, and RICAM, Linz, Austria (christian.schmeiser@univie.ac.at).
} 
for chemotaxis, derived by Patlak [25] and Keller and Segel [19, 20], we assume here that diffusion of the chemoattractant is fast compared to the characteristic time scales of the problem, which leads to an elliptic instead of a parabolic equation for $S$. More importantly, the chemotactic flux in Equation (1.1) decreases to zero as the cell density approaches a maximal value (here normalized to 1 ). In the classical model, the chemotactic flux is typically of the form $\varrho \nabla_{x} S$, allowing for concentration phenomena where the cell density becomes unbounded in finite time. A detailed review on results for the classical model can be found in [16] and [17]. A chemotaxis model of the type considered here (but with a parabolic equation for $S$ ) was first introduced by Hillen and Painter in [15], where the authors also showed that the additional volume-filling term in the cell flux leads to the global existence of solutions.

The paper at hand is strongly linked to [11], where model (1.1)-(1.4) is studied on the interval $(0, L)$, and to [8], where the limit $\varepsilon \rightarrow 0$ has been carried out for weak solutions globally in time. A discussion of similarities and differences in the behavior of the system depending on the space dimension will follow below.

Finally, we want to mention [4], where system (1.1), (1.2), (1.3) was investigated on unbounded domains, and its behavior was compared to that of a similar model featuring a nonlinear diffusion term. We also refer to this paper for a more extensive discussion of the literature.

The paper is organized as follows. In the first section, we briefly discuss properties of the solutions of the parabolic system (1.1)-(1.4) and of the associated hyperbolic system obtained by setting $\varepsilon=0$. In particular, we will show that under suitable conditions, solutions of the parabolic model converge to solutions of the hyperbolic system as $\varepsilon \rightarrow 0$. In contrast to the corresponding limit in the one-dimensional case, however (see [11]), this result is only valid for short times, i.e., before solutions of the hyperbolic model form shocks. We then complete the results obtained in [11] by showing the uniqueness of the vanishing viscosity limit for $d=1$.

Section 3 is dedicated to the asymptotic analysis of the parabolic model. We will see below that the dynamics of the problem in multiple space dimensions are driven by geometrical aspects that do not exist in one space dimension. Therefore it is not surprising that the characteristic time scales differ drastically, depending on $d=1$ or $d>1$. In the first case, typical time scales are of order $O\left(e^{-\frac{c}{\varepsilon}}\right)$, making it necessary to resolve exponentially small terms in the equations. Since matched asymptotic expansions fail here, an asymptotic projection method needs to be applied as was done in [11]. In contrast to this, typical time scales in the multi-dimensional problem are of order $O\left(\varepsilon^{-1}\right)$, and the long-time behavior of the system can be recovered by matched asymptotic expansions, as will be shown in section 3. As it turns out, this long-time behavior can be described as the motion of the interface between high and low density regions of $\varrho$. Properties of this interface motion are discussed in Section 4, and it is shown that the equation describing the motion of the interface falls into the class of surface diffusion models. We also discuss the stability of stationary solutions with respect to small perturbations. In particular, we investigate the question of whether the one-dimensional case can be attracting under certain conditions. Finally, in Section 5 , we will illustrate our theoretical results by performing numerical experiments for $d=2$ and 3 .

\section{Solutions properties for $\varepsilon>0$ and $\varepsilon=0$}

In this section, we will discuss certain properties of the solutions of (1.1)-(1.4) 
and the related hyperbolic chemotaxis model,

$$
\begin{aligned}
\partial_{t} \bar{\varrho}+\nabla_{x} \cdot\left(\bar{\varrho}(1-\bar{\varrho}) \nabla_{x} \bar{S}\right) & =0, \\
\Delta \bar{S} & =\bar{S}-\bar{\varrho},
\end{aligned}
$$

subject to

$$
\bar{\varrho}(x, 0)=\bar{\varrho}_{I}(x) \quad \text { in } \Omega \quad \text { and } \quad \partial_{\nu} \bar{S}=0 \quad \text { on } \partial \Omega .
$$

Let us start with some estimates for the parabolic problem.

Theorem 2.1. Let $\varrho_{I} \in L^{1}(\Omega)$, and $0 \leq \varrho_{I} \leq 1$. Then, there exists a unique, smooth solution of (1.1)-(1.4) that satisfies uniformly in $\varepsilon$

$$
(\varrho, S) \in L^{\infty}\left((0, \infty) ; L^{1}(\Omega) \times H^{1}(\Omega)\right)
$$

and

$$
0 \leq \varrho(x, t), S(x, t) \leq 1 \quad \text { and } \quad \int_{\Omega} \varrho d x=\int_{\Omega} S d x=\int_{\Omega} \varrho_{I} d x
$$

Proof. Local existence of smooth solutions can be shown by a standard contraction argument (see [11] for the one-dimensional case, or [4] for the corresponding results on unbounded domains in $\mathbb{R}^{d}$ ). The global existence then follows from a comparison principle. The estimates for $S$ are a direct consequence of (1.2).

For $d=1$, the existence of solutions of (2.1)-(2.3) follows from the convergence of solutions of the parabolic system in the limit of vanishing viscosity $\varepsilon \rightarrow 0$ (see [11]). In multiple space dimensions, proving the existence of hyperbolic solutions is by no means straightforward. The technical difficulties stem from the fact that no regularity estimates for the cell density are known in this case. Parallel to this work, a weak limit $\varepsilon \rightarrow 0$ has been carried out globally in time in [8], by employing a kinetic formulation of the problem. In the following, we prove a short time result of strong convergence of solutions of the parabolic problem to (smooth) solutions of (2.1)-(2.3).

TheOrEm 2.2. Let $\Omega \subset \mathbb{R}^{d}$, and let $(\varrho, S)$ be the solution of (1.1)-(1.4) with initial condition $\varrho_{I} \in H^{k}(\Omega), k>d / 2+1$, and $0 \leq \varrho_{I} \leq 1$, uniformly in $\varepsilon$. Then, as $\varepsilon \rightarrow 0$,

$$
\varrho \rightarrow \bar{\varrho} \text { in } C\left((0, T) ; H^{k-1}(\Omega)\right) \quad \text { and } S \rightarrow \bar{S} \text { in } C\left((0, T) ; C^{1}(\Omega)\right)
$$

for every $T<T^{*}$, with $T^{*}=\frac{1}{c} \ln \left(\frac{1+\left\|\varrho_{I}\right\|_{H^{k}(\Omega)}}{\left\|\varrho_{I}\right\|_{H^{k}(\Omega)}}\right)$, (迆, solves (2.1)-(2.3).

Proof. We apply $\nabla_{x}^{\alpha}$, with $|\alpha| \leq k$ to equation (1.1) and take the $L^{2}$-scalar product with $\nabla_{x}^{\alpha} \varrho$ to obtain

$$
\begin{aligned}
& \frac{d}{d t} \int_{\Omega}\left(\nabla_{x}^{\alpha} \varrho\right)^{2} d x \\
= & \varepsilon \int_{\Omega} \nabla_{x}^{\alpha} \Delta \varrho \nabla_{x}^{\alpha} \varrho d x-\int_{\Omega} \nabla_{x}^{\alpha} \varrho \nabla_{x}^{\alpha}\left((1-2 \varrho) \nabla_{x} \varrho \cdot \nabla_{x} S+\varrho(1-\varrho)(S-\varrho)\right) d x .
\end{aligned}
$$

Integration by parts of the first term and an application of Leibniz's formula

$$
\nabla_{x}^{\alpha}(u v)=\sum_{\beta \leq \alpha} c_{\alpha \beta} \nabla_{x}^{\beta} u \nabla_{x}^{\alpha-\beta} v
$$


where $\beta \leq \alpha$ means $\beta_{i} \leq \alpha_{i}, i=1, \ldots, d$, yields

$$
\begin{aligned}
\frac{d}{d t} \int_{\Omega}\left(\nabla_{x}^{\alpha} \varrho\right)^{2} d x \leq & -\int_{\Omega} \nabla_{x}^{\alpha} \varrho \sum_{\beta \leq \alpha} c_{\alpha \beta} \nabla_{x}^{\beta}\left[(1-2 \varrho) \nabla_{x} \varrho\right] \cdot \nabla_{x}^{\alpha-\beta} \nabla_{x} S d x \\
& -\int_{\Omega} \nabla_{x}^{\alpha} \varrho \sum_{\beta \leq \alpha} c_{\alpha \beta} \nabla_{x}^{\beta}[\varrho(1-\varrho)] \cdot \nabla_{x}^{\alpha-\beta}(S-\varrho) d x \\
= & I_{1}+I_{2} .
\end{aligned}
$$

We split the integral $I_{1}$ into two parts, thus separating the term with the highest derivative of $\varrho$ from the rest:

$$
\begin{aligned}
I_{1}:=I_{1,1}+I_{1,2}= & -\int_{\Omega} c_{\alpha \alpha} \nabla_{x}^{\alpha} \varrho \nabla_{x}^{\alpha}\left[(1-2 \varrho) \nabla_{x} \varrho\right] \cdot \nabla_{x} S d x \\
& -\int_{\Omega} \nabla_{x}^{\alpha} \varrho \sum_{\beta \leq \alpha} c_{\alpha \beta} \nabla_{x}^{\beta}\left[(1-2 \varrho) \nabla_{x} \varrho\right] \cdot \nabla_{x}^{\alpha-\beta} \nabla_{x} S d x
\end{aligned}
$$

where we sum over all $\beta \leq \alpha$ satisfying $|\beta|<|\alpha|$. Similarly, we split up $I_{1,1}$ by writing

$$
\begin{aligned}
I_{1,1} & =-\int_{\Omega} c_{\alpha \alpha} \nabla_{x}^{\alpha} \varrho\left[\sum_{\beta \leq \alpha} \nabla_{x}^{\beta}(1-2 \varrho) \nabla_{x}^{\alpha-\beta} \nabla_{x} \varrho\right] \cdot \nabla_{x} S d x \\
& =-\int_{\Omega} c_{\alpha \alpha} \nabla_{x}^{\alpha} \varrho\left[(1-2 \varrho) \nabla_{x}^{\alpha} \nabla_{x} \varrho \cdot \nabla_{x} S+\sum_{\beta \leq \alpha} \nabla_{x}^{\beta}(1-2 \varrho) \nabla_{x}^{\alpha-\beta} \nabla_{x} \varrho \cdot \nabla_{x} S\right] d x \\
& =I_{1,1,1}+I_{1,1,2}
\end{aligned}
$$

summing over all $\beta$ with $|\beta|>0$ in $I_{1,1,2}$. Since

$$
I_{1,1,1}=-\frac{1}{2} \int_{\Omega} c_{\alpha \alpha} \nabla_{x}\left(\nabla_{x}^{\alpha} \varrho\right)^{2}(1-2 \varrho) \cdot \nabla_{x} S d x,
$$

we can integrate by parts to obtain

$$
I_{1,1,1}=\frac{1}{2} \int_{\Omega} c_{\alpha \alpha}\left(\nabla_{x}^{\alpha} \varrho\right)^{2}\left((1-2 \varrho)(S-\varrho)-2 \nabla_{x} \varrho \cdot \nabla_{x} S\right) d x \leq c\|\varrho\|_{H^{k}(\Omega)}^{2}\left(1+\|\varrho\|_{H^{k}(\Omega)}\right) .
$$

Here we used the compact embedding of $H^{k}(\Omega)$ in $W^{1, \infty}(\Omega)$. Employing the inequality (see [28], Proposition 3.6, p. 9)

$$
\left\|\nabla_{x}^{\beta} f \nabla_{x}^{\alpha-\beta} g\right\|_{L^{2}(\Omega)} \leq c\left(\|f\|_{L^{\infty}(\Omega)}\|g\|_{H^{|\alpha|}(\Omega)}+\|f\|_{H^{|\alpha|}(\Omega)}\|g\|_{L^{\infty}(\Omega)}\right),
$$

we can estimate $I_{1,1,2}$ by

$$
I_{1,1,2} \leq c\left\|\nabla_{x}^{\alpha} \varrho\right\|_{L^{2}(\Omega)}\left(\left\|\nabla_{x} \varrho\right\|_{L^{\infty}(\Omega)}\|\varrho\|_{H^{k}(\Omega)}\right) \leq c\|\varrho\|_{H^{k}(\Omega)}^{3} .
$$

Analogously,

$$
I_{1,2} \leq c\left\|\nabla_{x}^{\alpha} \varrho\right\|_{L^{2}(\Omega)}\left(\left\|\nabla_{x} \varrho\right\|_{L^{\infty}(\Omega)}\|S\|_{H^{k+1}(\Omega)}+\|\varrho\|_{H^{k}(\Omega)}\left\|\nabla_{x}^{2} S\right\|_{L^{\infty}(\Omega)}\right) \leq c\|\varrho\|_{H^{k}(\Omega)}^{3},
$$

using again the embedding of $H^{k}(\Omega)$ and the fact that solutions of the elliptic Equation (1.2) fulfill the estimate $\|S\|_{H^{k+2}(\Omega)} \leq c\|\varrho\|_{H^{k}(\Omega)}$. Similarly, we can estimate $I_{2}$ by

$$
I_{2} \leq c\|\varrho\|_{H^{k}(\Omega)}^{2}
$$


Finally, adding over all multi-indices $|\alpha| \leq k$, we obtain

$$
\frac{d}{d t}\|\varrho\|_{H^{k}(\Omega)} \leq c\|\varrho\|_{H^{k}(\Omega)}\left(1+\|\varrho\|_{H^{k}(\Omega)}\right),
$$

and hence, $\varrho \in L^{\infty}\left((0, T) ; H^{k}(\Omega)\right)$ for every $T<T^{*}$. This, together with the fact that $\partial_{t} \varrho \in L^{2}((0, T) \times \Omega)$, implies that $\varrho$ lies in a compact set in the space $C\left((0, T) ; H^{k-1}(\Omega)\right.$ ) (see [27]). From the uniform estimates for $\varrho$, it can be easily shown that $\partial_{t} \nabla_{x} S \in L^{2}((0, T) \times \Omega)$ and $\nabla_{x}^{2} S \in L^{\infty}((0, T) \times \Omega)$ for $T<T^{*}$. Then, it follows from an anisotropic generalization of the Sobolev embedding of $W^{1, p}$ in $C^{0,1-n / p}, p>n$ (see [14]) that $\nabla_{x} S$ is uniformly bounded in the space $C^{0, \beta}([0, T] \times \Omega$ ), $0<\beta \leq 1 / 3$, implying the assertion.

In [8] it has been shown that (2.1)-(2.3) possesses global entropy solutions satisfying the following conditions:

(i) $\varrho \in L^{\infty}((0, \infty) \times \Omega)$ and, consequently, $S \in L^{\infty}\left((0, \infty), W^{2, p}(\Omega)\right)$ for all $p<\infty$.

(ii) For all convex entropies $\eta$ and entropy fluxes $q$ such that $q^{\prime}(\varrho)=(1-2 \varrho) \eta^{\prime}(\varrho)$,

$$
\partial_{t} \eta(\varrho)+\nabla_{x} \cdot\left(q(\varrho) \nabla_{x} S\right)+(\varrho-S)\left(q(\varrho)-\varrho(1-\varrho) \eta^{\prime}(\varrho)\right) \geq 0,
$$

in the sense of distributions with initial data $\eta\left(\varrho_{I}\right)$.

We will now complete the results in [11] by proving the uniqueness of entropy solutions of (2.1)-(2.3) in one space dimension. To this end, we will apply results from the theory of nonlinear degenerate parabolic problems, of which the model can be seen as a special case:

THEOREM 2.3. Let $\Omega \subset \mathbb{R}$, and let $\bar{\varrho}(x, t)$ be an entropy solution of (2.1)-(2.3) with initial condition $\bar{\varrho}_{I} \in B V(\Omega), 0 \leq \bar{\varrho}_{I} \leq 1$. Then, this entropy solution is unique in the space $L^{\infty}((0, T), B V(\Omega))$.

Proof. The proof can be carried out analogously to the proof of Theorem 3.20 in [4]: let $\bar{\varrho}^{1}, \bar{\varrho}^{2}$ be two different entropy solutions of (2.1), (2.2) with initial data $\bar{\varrho}_{I}^{1}$ and $\bar{\varrho}_{I}^{2}$. Then, it follows from [18], Theorem 1.3 that these solutions satisfy the estimate

$$
\begin{gathered}
\left\|\bar{\varrho}^{1}(\cdot, t)-\bar{\varrho}^{2}(\cdot, t)\right\|_{L^{1}(\Omega)} \leq\left\|\bar{\varrho}_{I}^{1}-\bar{\varrho}_{I}^{2}\right\|_{L^{1}(\Omega)}+ \\
c_{1} t\left\|\bar{S}_{x}^{1}-\bar{S}_{x}^{2}\right\|_{L^{\infty}((0, T) ; B V(\Omega))}+c_{2} t\left\|\bar{S}_{x}^{1}-\bar{S}_{x}^{2}\right\|_{L^{\infty}((0, T) \times \Omega)},
\end{gathered}
$$

with $\bar{S}^{1}$ and $\bar{S}^{2}$ satisfying (2.2) with $\bar{\varrho}^{1}$ and $\bar{\varrho}^{2}$, respectively. Since there exists a constant $c>0$ such that

$$
\left\|\bar{S}_{x}^{1}-\bar{S}_{x}^{2}\right\|_{L^{\infty}((0, T) ; B V(\Omega))} \leq c\left\|\varrho^{1}-\bar{\varrho}^{2}\right\|_{L^{1}(\Omega)}
$$

and

$$
\left\|\bar{S}_{x}^{1}-\bar{S}_{x}^{2}\right\|_{L^{\infty}((0, T) \times(\Omega))} \leq c\left\|\bar{\varrho}^{1}-\bar{\varrho}^{2}\right\|_{L^{1}(\Omega)},
$$

we can estimate the right hand side only in terms of $\left\|\varrho^{1}-\bar{\varrho}^{2}\right\|_{L^{1}(\Omega)}$, eventually leading to the conclusion that if $\bar{\varrho}_{I}^{1}=\bar{\varrho}_{I}^{2}$ a.e., then also $\bar{\varrho}^{1}=\bar{\varrho}^{2}$ a.e. for $0 \leq t \leq T$.

Next, we give an explicit expression for the convergence rate as $\varepsilon \rightarrow 0$ : 
ThEOREM 2.4. Let $\Omega \subset \mathbb{R}$, and let $\varrho(x, t)$ and $\bar{\varrho}(x, t)$ be the unique (entropy) solutions of (1.1)-(1.4) and (2.1)-(2.3), respectively. Then, there exist constants $c_{1}, c_{2}>0$ such that for time $t<c_{1}^{-1}$, the following estimate holds:

$$
\|\varrho(\cdot, t)-\bar{\varrho}(\cdot, t)\|_{L^{1}(\Omega)} \leq \frac{c_{2}}{1-c_{1} t} \sqrt{\varepsilon t} .
$$

Proof. The proof can be carried out analogously to the proof of Theorem 1.1 of [13], where the continuous dependence of entropy solutions of nonlinear, degenerate parabolic equations on the flux and the diffusion function is proven. In particular, it follows from the results in [13] that the solutions $\varrho$ and $\varrho$ satisfy the estimate

$$
\begin{gathered}
\|\varrho(\cdot, t)-\bar{\varrho}(\cdot, t)\|_{L^{1}(\Omega)} \leq\left\|\varrho_{I}(\cdot)-\bar{\varrho}_{I}(\cdot)\right\|_{L^{1}(\Omega)}+ \\
\bar{c}_{1} t\left(\left\|\bar{S}_{x}^{1}-\bar{S}_{x}^{2}\right\|_{L^{\infty}((0, T) \times \Omega)}+\left\|\bar{S}_{x}^{1}-\bar{S}_{x}^{2}\right\|_{L^{\infty}((0, T) ; B V(\Omega))}\right)+\bar{c}_{2} \sqrt{\varepsilon t} .
\end{gathered}
$$

Choosing initial conditions $\varrho_{I}=\bar{\varrho}_{I}$ a.e. and again using estimates $(2.8)$ and (2.9), the assertion follows for $t<c_{1}^{-1}$.

As was shown in [11] for one space dimension, the long-time behavior of the hyperbolic system (2.1)-(2.3) differs significantly from that of the parabolic system (1.1)-(1.4), so that an estimate that is non-uniform in time is actually the best result to be expected. As we will see, the asymptotic behavior depends also in multiple space dimensions strongly on whether $\varepsilon=0$ or $\varepsilon>0$. While we will investigate the latter case in the next chapter, we conclude this section with a short discussion of stationary solutions for $\varepsilon=0$.

Looking at the stationary version of (2.1), (2.2), it can be anticipated that nonconstant stationary solutions consist of patches where $\bar{\varrho}$ is either zero or one. Indeed, we can define a whole manifold of stationary entropy solutions $\left(\bar{\varrho}_{\infty}, \bar{S}_{\infty}\right)$ of $(2.1)-(2.3)$, given by

$$
\bar{\varrho}_{\infty}(x)=\left\{\begin{array}{ll}
1 & \text { in } \bar{\Omega} \\
0 & \text { else },
\end{array} \quad \text { and } \quad \Delta \bar{S}_{\infty}=\bar{\varrho}_{\infty}-\bar{S}_{\infty}\right.
$$

together with Neumann boundary conditions $(2.3)$ for $\bar{S}_{\infty}$. Each of these solutions is characterized by the shape of the domain $\bar{\Omega} \subset \Omega$ and subject to the entropy condition (2.7). An alternative formulation of the entropy condition for solutions of type (2.10) is given by

LEMma 2.5. Let $\left(\bar{\varrho}_{\infty}, \bar{S}_{\infty}\right)$ be a stationary entropy solution of system (2.1)-(2.3) satisfying (2.10) with a Lipschitz domain $\bar{\Omega} \subset \Omega$. Then, we have that $\partial_{n} \bar{S}_{\infty} \leq 0$ on $\partial \bar{\Omega} \backslash \partial \Omega, n$ being the unit outer normal on $\partial \bar{\Omega}$.

Proof. Fixing a constant $\alpha \in(0,1)$ and splitting the integral into the regions where $\bar{\varrho}_{\infty}=0$ and where $\bar{\varrho}_{\infty}=1$, we can write $(2.7)$ as

$$
\begin{aligned}
-\int_{0}^{\infty} & \int_{\bar{\Omega}}\left[\alpha(1-\alpha) \nabla_{x} \bar{S}_{\infty} \cdot \nabla_{x} \varphi+\alpha(1-\alpha) \Delta \bar{S}_{\infty} \varphi\right] d x d t \\
& +\int_{0}^{\infty} \int_{\Omega \backslash \bar{\Omega}}\left[\alpha(1-\alpha) \nabla_{x} \bar{S}_{\infty} \cdot \nabla_{x} \varphi+\alpha(1-\alpha) \Delta \bar{S}_{\infty} \varphi\right] d x d t \geq 0,
\end{aligned}
$$


where the time derivatives in (2.7) vanish due to the fact that $\left(\bar{\varrho}_{\infty}, \bar{S}_{\infty}\right)$ is a stationary solution. Arranging terms differently, we obtain

$$
\alpha(1-\alpha)\left[\int_{0}^{\infty} \int_{\bar{\Omega}} \nabla \cdot\left(\varphi \nabla_{x} \bar{S}_{\infty}\right) d x d t-\int_{0}^{\infty} \int_{\Omega \backslash \bar{\Omega}} \nabla_{x} \cdot\left(\varphi \nabla_{x} \bar{S}_{\infty}\right) d x d t\right] \leq 0,
$$

yielding, after an application of the divergence theorem,

$$
\alpha(1-\alpha) \int_{0}^{\infty} \int_{\partial \bar{\Omega} \backslash \partial \Omega} \varphi \partial_{n} \bar{S}_{\infty} d \sigma d t \leq 0 .
$$

Since this inequality holds for arbitrary test functions $\varphi(x, t)$, the assertion follows.

3. Asymptotic behavior of the parabolic problem

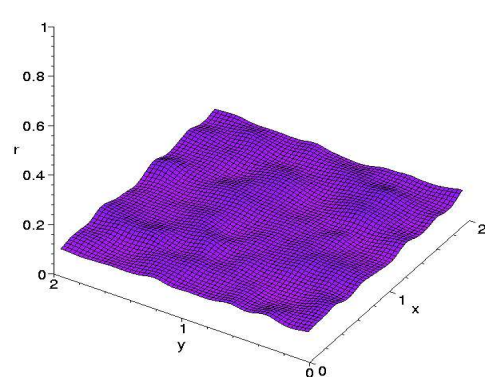

(a) $t=33$

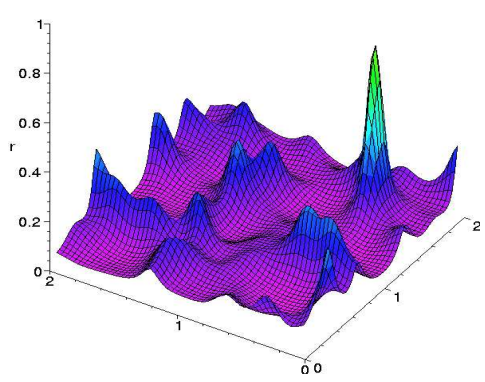

(b) $t=58$

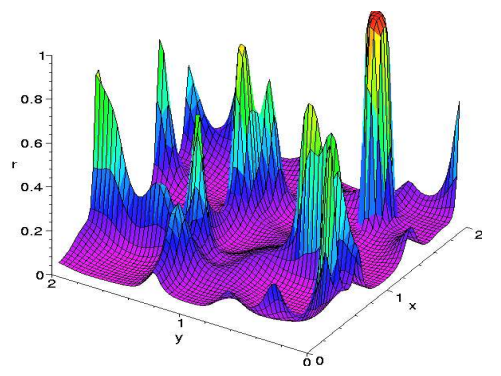

(c) $t=65$

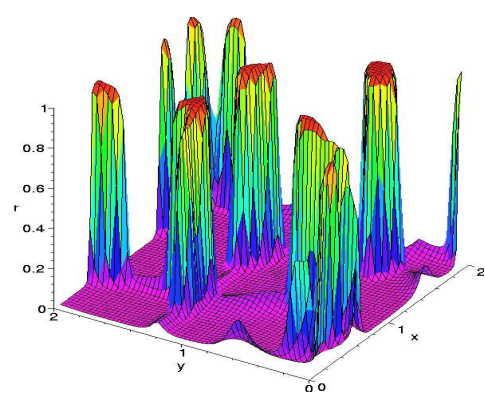

(d) $t=84$

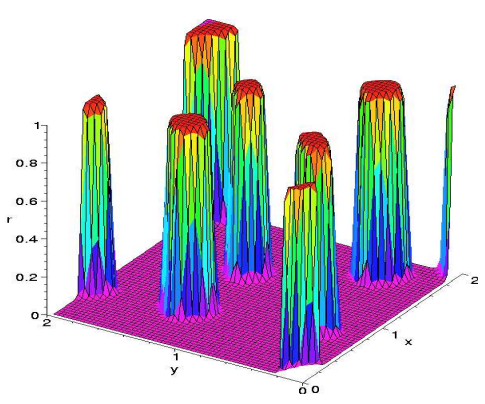

(e) $t=250$

FIG. 3.1. Numerical solution of the parabolic system (1.1)-(1.4) with random initial data $\varrho_{I} \in[0.1,0.11]$ and $\varepsilon=10^{-4}$.

Let us motivate our analysis on the long-time behavior of system (1.1)-(1.4) for $d>1$ by a numerical study. Figure 3.1 shows a typical evolution of the system in two 
space dimensions, where we chose a random perturbation of a spatially homogeneous background as initial condition. It can be observed that by the attracting effect of the chemical, the random perturbations of the cell density are amplified and aggregations start to form. Since more chemical is produced where cells have already crowded, the regions between the aggregations start to empty, and a distinct pattern of high density and vacuum regions emerges. Due to the density control in the chemotactic flux the cell density remains bounded, and the system evolves to a pattern where $\varrho$ is either almost zero or almost one, with a sharp but smooth transition between the two phases. Once this state has been reached it remains by no means stationary. Regions of high cell density attract each other and move slowly towards each other or towards the boundary (see also the numerical experiments in Section 5). This behavior has also been observed in models with a parabolic equation for $S[26,15]$.

The movement of high-density regions takes place on a time scale that is much larger than the 'hyperbolic' time scale on which the formation and merging of peaks can be observed. Hence, we rescale time $t \rightarrow t / \varepsilon$ in Equation (1.1), leading to the rescaled system

$$
\begin{aligned}
\varepsilon \partial_{t} \varrho & =\nabla_{x} \cdot\left(\varepsilon \nabla_{x} \varrho-\varrho(1-\varrho) \nabla_{x} S\right) \\
\Delta S & =S-\varrho .
\end{aligned}
$$

Our aim is to investigate the slow dynamics of a solution that is, for every moment in time, close to one of the stationary entropy solutions defined by (2.10). Therefore, we look for solutions whose limit $\left(\bar{\varrho}_{0}, S_{0}\right)$ as $\varepsilon \rightarrow 0$ satisfies

$$
\bar{\varrho}_{0}(x, t)=\left\{\begin{array}{ll}
1 & \text { in } \bar{\Omega}(t) \\
0 & \text { else, }
\end{array} \quad \text { and } \quad \Delta S_{0}=\bar{\varrho}_{0}-S_{0},\right.
$$

with Neumann boundary conditions for $S_{0}$. For the cell density the limit has to be understood away from a boundary layer around the free boundary $\Gamma(t):=\partial \bar{\Omega}(t) \backslash \partial \Omega$, which we assume to be smooth. We also assume that the entropy condition from Lemma 2.5 is satisfied in the strict sense, i.e.,

$$
\partial_{n} S_{0}<0 \quad \text { for } \quad x \in \Gamma(t) .
$$

The asymptotics below will provide a description of the dynamics of $\Gamma(t)$.

We introduce a new, local coordinate system defined by the normal and the tangential directions on the interface: let $n$ be the unit outward normal on $\Gamma(t)$, and let $\tau_{i}$ be a set of locally orthogonal unit vectors tangential to $\Gamma(t)$. We define $z=z(x, t) \in \mathbb{R}$ as the signed distance to the interface oriented along $n$ (i.e., $z<0$ in $\bar{\Omega}(t)$ and $z>0$ in $\Omega \backslash \bar{\Omega}(t))$. Moreover, we define $\sigma=\sigma(x, t) \in \mathbb{R}^{d-1}$ as a vector whose components $\sigma_{i}$ are the arc lengths of curves oriented according to the $\tau_{i}$. Denoting points on the interface by $\Gamma(t)=\{X(\sigma, t)\}$, we can express every point $x \in \Omega$ that is sufficiently close to the interface as a function of $z$ and $\sigma$, i.e., $x=X(\sigma, t)+z n(\sigma, t)$. The Jacobian of the inverse map is given by (see for instance [1]; for a more detailed derivation for this particular case see also [6])

$$
\nabla_{x} z=n, \quad \nabla_{x} \sigma_{i}=\frac{\partial_{\sigma_{i}} X}{1+z\left(\partial_{\sigma_{i}} n \cdot \partial_{\sigma_{i}} X\right)} .
$$


Writing $\varrho$ as a function of the new variables $\sigma$ and $z$, equation (3.1) transforms into

$$
\begin{aligned}
& \varepsilon\left(\partial_{t} \varrho+\sum_{i=1}^{d-1} \partial_{\sigma_{i}} \varrho \partial_{t} \sigma_{i}+\partial_{z} \varrho \partial_{t} z\right)+\left(\sum_{i=1}^{d-1} \nabla_{x} \sigma_{i} \partial_{\sigma_{i}}+\nabla_{x} z \partial_{z}\right) \\
& \times\left[\varrho(1-\varrho)\left(\sum_{j=1}^{d-1} \nabla_{x} \sigma_{j} \partial_{\sigma_{j}} S+\nabla_{x} z \partial_{z} S\right)-\varepsilon\left(\sum_{j=1}^{d-1} \nabla_{x} \sigma_{j} \partial_{\sigma_{j}} \varrho+\nabla_{x} z \partial_{z} \varrho\right)\right]=0 .
\end{aligned}
$$

As a next step, we introduce the layer variable $\xi=z / \varepsilon$ and make the ansatz

$$
\varrho=\hat{\varrho}_{0}(\sigma, \xi, t)+\varepsilon \hat{\varrho}_{1}(\sigma, \xi, t)+O\left(\varepsilon^{2}\right) .
$$

The boundary layer terms have to satisfy the matching conditions

$$
\hat{\varrho}_{0}(\sigma, \infty, t)=1, \quad \hat{\varrho}_{0}(\sigma,-\infty, t)=0, \quad \hat{\varrho}_{1}(\sigma, \pm \infty, t)=0 .
$$

The boundary layer terms in the cell density are expected to only contribute terms of order $O\left(\varepsilon^{2}\right)$ to the chemical density, i.e., we make the ansatz

$$
S(\sigma, z, t)=S_{0}(\sigma, z, t)+O\left(\varepsilon^{2}\right),
$$

with $S_{0}$ as given in (3.3). Since the chemical density $S$ varies only slowly across the interface, we obtain

$$
\begin{aligned}
S(\sigma, z, t) & =S_{0}(\sigma, \varepsilon \xi, t)+O\left(\varepsilon^{2}\right) \\
& =S_{0}(\sigma, 0, t)+\varepsilon \xi \partial_{n} S_{0}(\sigma, 0, t)+O\left(\varepsilon^{2}\right) .
\end{aligned}
$$

Here the notation $\partial_{n} S_{0}$ was used instead of $\partial_{z} S_{0}$ for consistency.

We continue by solving the layer equations. To this end, we must determine $\nabla_{x} \sigma_{i}$ as a function of $\xi$ and compute the dot products between $\nabla_{x} \sigma_{i}, \nabla_{x} z$ and their derivatives with respect to $\sigma_{i}$ and $\xi$. From (3.5), it follows that

$$
\begin{aligned}
\nabla_{x} \sigma_{i} & =\partial_{\sigma_{i}} X\left(1+\varepsilon \xi\left(\partial_{\sigma_{i}} n \cdot \partial_{\sigma_{i}} X\right)\right)^{-1} \\
& =\partial_{\sigma_{i}} X\left(1-\varepsilon \xi\left(\partial_{\sigma_{i}} n \cdot \partial_{\sigma_{i}} X\right)+\varepsilon^{2} \xi^{2}\left(\partial_{\sigma_{i}} n \cdot \partial_{\sigma_{i}} X\right)^{2}+O\left(\varepsilon^{3}\right)\right) .
\end{aligned}
$$

Thus,

$$
\begin{gathered}
\nabla_{x} \sigma_{i} \cdot \partial_{\sigma_{i}} \nabla_{x} \sigma_{i}=O(\varepsilon), \quad \nabla_{x} \sigma_{i} \cdot \partial_{\sigma_{i}} \nabla_{x} \sigma_{j}=0, \quad \nabla_{x} \sigma_{i} \cdot \partial_{\sigma_{i}} \nabla_{x} z=\partial_{\sigma_{i}} n \cdot \partial_{\sigma_{i}} X+O(\varepsilon), \\
\nabla_{x} z \cdot \partial_{\xi} \nabla_{x} \sigma_{i}=0, \quad \nabla_{x} z \cdot \partial_{\xi} \nabla_{x} z=0 .
\end{gathered}
$$

Now using the expansion for $\varrho$, Equation (3.6) becomes

$$
\begin{aligned}
& \varepsilon \partial_{t} \hat{\varrho}_{0}+\varepsilon \sum_{i=1}^{d-1} \partial_{\sigma_{i}} \hat{\varrho}_{0} \partial_{t} \sigma_{i}+\partial_{\xi} \hat{\varrho}_{0} \partial_{t} z+\varepsilon \partial_{\xi} \hat{\varrho}_{1} \partial_{t} z \\
& +\left(\sum_{i=1}^{d-1} \nabla_{x} \sigma_{i} \partial_{\sigma_{i}}+\frac{1}{\varepsilon} \nabla_{x} z \partial_{\xi}\right) \cdot\left[( \hat { \varrho } _ { 0 } ( 1 - \hat { \varrho } _ { 0 } ) + \varepsilon \hat { \varrho } _ { 1 } ( 1 - 2 \hat { \varrho } _ { 0 } ) ) \left(\sum_{j=1}^{d-1} \nabla_{x} \sigma_{j}\left(\partial_{\sigma_{j}} S_{0}+\varepsilon \xi \partial_{\sigma_{j}} \partial_{n} S_{0}\right)\right.\right. \\
& \left.\left.+\nabla_{x} z\left(\partial_{n} S_{0}+\varepsilon \xi \partial_{n}^{2} S_{0}\right)\right)-\varepsilon\left(\sum_{j=1}^{d-1} \nabla_{x} \sigma_{j} \partial_{\sigma_{j}} \hat{\varrho}_{0}+\frac{1}{\varepsilon} \nabla_{x} z \partial_{\xi} \hat{\varrho}_{0}+\nabla_{x} z \partial_{\xi} \hat{\varrho}_{1}\right)\right]=O(\varepsilon)
\end{aligned}
$$


where $S_{0}$ is always evaluated at $(\sigma, 0, t)$. Comparing terms of the order $O\left(\varepsilon^{-1}\right)$ in $(3.7)$ gives

$$
\partial_{\xi}\left(\hat{\varrho}_{0}\left(1-\hat{\varrho}_{0}\right) \partial_{n} S_{0}-\partial_{\xi} \hat{\varrho}_{0}\right)=0
$$

and, by integration and the matching conditions,

$$
\partial_{\xi} \hat{\varrho}_{0}=\hat{\varrho}_{0}\left(1-\hat{\varrho}_{0}\right) \partial_{n} S_{0}
$$

The solution of (3.8) can only be determined up to a shift in $\xi$, which is fixed uniquely by $\hat{\varrho}_{0}(\sigma, 0, t)=\frac{1}{2}$.

Proceeding to the $O(1)$-terms in (3.7), we obtain

$$
\begin{aligned}
\partial_{\xi} \hat{\varrho}_{0} \partial_{t} z & +\sum_{i=1}^{d-1} \partial_{\sigma_{i}}\left(\hat{\varrho}_{0}\left(1-\hat{\varrho}_{0}\right) \partial_{\sigma_{i}} S_{0}\right)+\left(\partial_{\sigma_{i}} n \cdot \partial_{\sigma_{i}} X\right)\left(\hat{\varrho}_{0}\left(1-\hat{\varrho}_{0}\right) \partial_{n} S_{0}-\partial_{\xi} \hat{\varrho}_{0}\right) \\
& +\partial_{\xi}\left(\hat{\varrho}_{0}\left(1-\hat{\varrho}_{0}\right) \xi \partial_{n}^{2} S_{0}+\hat{\varrho}_{1}\left(1-2 \hat{\varrho}_{0}\right) \partial_{n} S_{0}-\partial_{\xi} \hat{\varrho}_{1}\right)=0 .
\end{aligned}
$$

Thanks to (3.8), the third term vanishes. Integrating (3.9) with respect to $\xi$ from $-\infty$ to $+\infty$ and using the fact that

$$
\int_{-\infty}^{\infty} \hat{\varrho}_{0}\left(1-\hat{\varrho}_{0}\right) d \xi=\int_{-\infty}^{\infty} \frac{\partial_{\xi} \hat{\varrho}_{0}}{\partial_{n} S_{0}} d \xi=-\frac{1}{\partial_{n} S_{0}},
$$

yields the solvability condition for $\hat{\varrho}_{1}$, i.e.,

$$
\partial_{t} z+\sum_{i=1}^{d-1} \partial_{\sigma_{i}}\left(\frac{\partial_{\sigma_{i}} S_{0}}{\partial_{n} S_{0}}\right)=0
$$

Since $V_{n}=-\partial_{t} z$ is the normal velocity of $\Gamma(t)$, this is the desired description of the dynamics of $\bar{\Omega}(t)$. We collect the results of this section:

Lemma 3.1. Assume there is a solution of (3.1), (3.2), whose limit as $\varepsilon \rightarrow 0$ satisfies (3.3) away from the smooth free boundary $\Gamma(t)=\partial \bar{\Omega}(t) \backslash \partial \Omega$. Assume further that in a neighborhood of the free boundary the cell density has an asymptotic expansion of the form $\varrho=\hat{\varrho}_{0}(\sigma, z / \varepsilon, t)+\varepsilon \hat{\varrho}_{1}(\sigma, z / \varepsilon, t)+O\left(\varepsilon^{2}\right)$, where $\sigma$ and $z$ are tangential and orthogonal coordinates as introduced above, and $\hat{\varrho}_{0}$ and $\hat{\varrho}_{1}$ are $\varepsilon$-independent, smooth, and bounded. Then the dynamics of the free boundary is determined by

$$
\begin{array}{ll}
V_{n}=\nabla_{\sigma} \cdot\left(\frac{1}{\partial_{n} S} \nabla_{\sigma} S\right) & \text { on } \Gamma(t)=\partial \bar{\Omega}(t) \backslash \partial \Omega, \\
-\Delta S+S=\chi_{\bar{\Omega}(t)} & \text { in } \Omega, \quad \partial_{\nu} S=0 \text { on } \partial \Omega
\end{array}
$$

\section{Properties of the interface motion}

In this section we investigate some of the formal properties of the geometric flow model (3.11) for the interface. We start with a geometric property. Consider a situation where the interface $\Gamma(t)$ meets a straight part of the domain boundary $\partial \Omega$. Since $S$ satisfies homogeneous Neumann boundary conditions, a local reflection of the solution with respect to $\partial \Omega$ extends the solution to the exterior of $\Omega$. Assuming that the interface dynamics has a smoothing effect on $\Gamma(t)$ (see below for arguments supporting this assumption), we conclude that $\Gamma(t)$ meets $\partial \Omega$ orthogonally. Actually, 
the argument can also be applied to curved parts of $\partial \Omega$ after an appropriate coordinate transformation straightening out the boundary. Thus, we conclude that

$$
\Gamma(t) \perp \partial \Omega, \quad \text { i.e., } \quad n \cdot \nu=0,
$$

at points of intersection. These points constitute the boundary $\partial \Gamma(t)$ of the interface, and by the Neumann boundary conditions for $S$, the normal component $\partial_{\nu} S / \partial_{n} S$ of the surface diffusion flux in (3.11) vanishes along $\partial \Gamma(t)$. As a consequence, the mass conservation property of the original model carries over to (3.11), where it takes the form of conservation of the volume of $\bar{\Omega}(t)$ :

$$
\frac{d}{d t} \int_{\bar{\Omega}(t)} d x=\int_{\Gamma(t)} V_{n} d \sigma=0
$$

where the identity for the time derivative of the volume follows from Theorem 4.2 in [10, p. 352]. Actually, a stronger conservation property holds. Introducing the splitting $\Gamma(t)=\bigcup_{i=1}^{N} \Gamma_{i}(t)$ of the free boundary into its connected components $\Gamma_{1}(t), \ldots, \Gamma_{N}(t)$, the integral of $V_{n}$ over each $\Gamma_{i}(t)$ vanishes, implying that the volume of each connected component of $\bar{\Omega}(t)$ (and of $\Omega \backslash \bar{\Omega}(t)$ ) is preserved as long as the topology of $\bar{\Omega}(t)$ does not change.

4.1. Gradient flow structure. The interface motion problem possesses a gradient flow structure with respect to the energy

$$
\hat{E}[\bar{\Omega}]=-\frac{1}{2} \int_{\bar{\Omega}} S[\bar{\Omega}] d x
$$

where $S[\bar{\Omega}] \in H^{1}(\Omega)$ is the unique solution of

$$
\int_{\Omega}(\nabla S \cdot \nabla \varphi+S \varphi) d x=\int_{\bar{\Omega}} \varphi d x \quad \forall \varphi \in H^{1}(\Omega) .
$$

Note that the choice $\varphi=S$ leads to an alternative representation of the energy:

$$
\hat{E}[\bar{\Omega}]=-\frac{1}{2} \int_{\Omega}\left(|\nabla S[\bar{\Omega}]|^{2}+S[\bar{\Omega}]^{2}\right) d x .
$$

In the following we take a closer look on shape sensitivities of the energy functional. However, since the dynamics operates on families of splittings of $\Omega$ into subdomains with fixed volume, a parametrization of such families is needed. The shape sensitivities are then computed as derivatives of the energy with respect to the parameters. We shall derive such a parametrization in the neighborhood of a shape $\bar{\Omega}_{0}$ whose free boundary is given by $\Gamma_{0}=\partial \bar{\Omega}_{0} \backslash \partial \Omega=\bigcup_{i=1}^{N} \Gamma_{0 i}$ with connected components $\Gamma_{01}, \ldots, \Gamma_{0 N}$ written as $\Gamma_{0 i}=\left\{X(\sigma): \sigma \in P_{i}\right\}$. The free boundary of a neighbouring shape $\widetilde{\Omega}$ can then be written as

$$
\widetilde{\Gamma}=\bigcup_{i=1}^{N} \widetilde{\Gamma}_{i} \quad \text { with } \quad \widetilde{\Gamma}_{i}=\left\{X(\sigma)+n(\sigma) V(\sigma): \sigma \in P_{i}\right\} .
$$

The signed volume between $\Gamma_{0 i}$ and $\widetilde{\Gamma}_{i}$ can be computed as

$$
\int_{\Gamma_{0 i}}\left(V+\frac{\kappa V^{2}}{2}\right) d \sigma, \quad i=1, \ldots, N
$$


where $\kappa$ denotes the signed mean curvature along $\Gamma_{0 i}$ (oriented such that $\kappa<0$ on convex and $\kappa>0$ on concave parts). The shapes $\bar{\Omega}_{0}$ and $\widetilde{\Omega}$ belong to the same family if all the integrals in (4.5) vanish. The family will be parametrized by functions $W(\sigma)$ with $\int W d \sigma_{i}=0, i=1, \ldots, N$, where here and in the following $d \sigma_{i}$ denotes the restriction of the surface measure $d \sigma$ to $\Gamma_{0 i}$. For the construction, fixed functions $W_{0 i}(\sigma)$ will be needed, which satisfy

$$
\int W_{0 i} d \sigma_{i}=1
$$

Setting $V[W]=W+A_{i}[W] W_{0 i}$ on $\Gamma_{0 i}$, the constant

$$
A_{i}[W]=\frac{-\int \kappa W^{2} d \sigma_{i}}{1+\int \kappa W_{0 i} W d \sigma_{i}+\sqrt{\left(1+\int \kappa W_{0 i} W d \sigma_{i}\right)^{2}-\int \kappa W_{0 i}^{2} d \sigma_{i} \int \kappa W^{2} d \sigma_{i}}}
$$

is chosen such that it solves

$$
A_{i}^{2} \int \kappa W_{0 i}^{2} d \sigma_{i}+2 A_{i}\left(1+\int \kappa W_{0 i} W d \sigma_{i}\right)+\int \kappa W^{2} d \sigma_{i}=0,
$$

which is equivalent to the requirement that the integrals in (4.5) vanish. Note that, for small values of $W$,

$$
A_{i}[W]=-\int \frac{\kappa W^{2}}{2} d \sigma_{i}+O\left(W^{3}\right),
$$

and, in particular, the linearization of $V[W]$ at $W=0$ is the identity.

The energy is now evaluated for all shapes $\bar{\Omega}[W]$ which are determined by their free boundaries

$$
\begin{gathered}
\Gamma[W]=\bigcup_{i=1}^{N} \Gamma_{i}[W] \quad \text { with } \Gamma_{i}[W]=\left\{X(\sigma)+n(\sigma) V[W](\sigma): \sigma \in P_{i}\right\}, \\
E[W]:=\hat{E}[\bar{\Omega}[W]]=-\frac{1}{2} \int_{\bar{\Omega}[W]} S[W] d x
\end{gathered}
$$

with the abbreviation $S[\bar{\Omega}[W]] \rightarrow S[W]$. For computing variations of $E$, the Frechet derivative $S^{\prime}[W]$ will be needed. From the weak formulation of the problem for $S[W]$

$$
\int_{\Omega}(\nabla S \cdot \nabla \varphi+S \varphi) d x=\int_{\bar{\Omega}[W]} \varphi d x
$$

the problem

$$
\int_{\Omega}\left(\nabla S^{\prime} \cdot \nabla \varphi+S^{\prime} \varphi\right) d x=\int \varphi(X+n V[W])(1+\kappa V[W]) V^{\prime}[W](\hat{W}) d \sigma
$$

for $S^{\prime}[W](\hat{W})$ is easily computed. A similar computation is needed for the first variation of the energy:

$$
\begin{aligned}
E^{\prime}[W](\hat{W})= & -\frac{1}{2} \int_{\bar{\Omega}[W]} S^{\prime}[W](\hat{W}) d x \\
& -\frac{1}{2} \int S[W](X+n V[W])(1+\kappa V[W]) V^{\prime}[W](\hat{W}) d \sigma .
\end{aligned}
$$


Setting $\varphi=S^{\prime}$ in (4.7) and $\varphi=S$ in (4.8) shows that the two terms on the right hand side are equal, and thus,

$$
E^{\prime}[W](\hat{W})=-\int S[W](X+n V[W])(1+\kappa V[W]) V^{\prime}[W](\hat{W}) d \sigma .
$$

In particular, the first variation evaluated at the reference shape $\bar{\Omega}[0]$ is given by

$$
E^{\prime}[0](W)=-\int S[0] W d \sigma,
$$

since $V^{\prime}[0](W)=W$, as mentioned above.

From the form (4.10) of the shape derivative one observes that the shape gradient (in the $L^{2}$-metric on the surface) can be identified with $-S$. On the other hand, for fixed $\partial_{n} S<0$ and for $W \in H^{-1}(\Gamma)$ with $\int W d \sigma_{i}=0, i=1, \ldots, N$, we define $g(W) \in$ $H^{1}(\Gamma)$ by

$$
\nabla_{\sigma} \cdot\left(\frac{1}{\partial_{n} S} \nabla_{\sigma} g(W)\right)=W
$$

The map $g$ is positive definite, and a weighted $H^{-1}(\Gamma)$-scalar product can be defined by

$$
\left\langle W_{1}, W_{2}\right\rangle:=\int \frac{\nabla_{\sigma} g\left(W_{1}\right) \cdot \nabla_{\sigma} g\left(W_{2}\right)}{-\partial_{n} S} d \sigma
$$

The following result follows immediately by comparison with (4.10).

LEMMA 4.1. The linearized geometric flow model (3.11) (with frozen $\partial_{n} S$ ) can be interpreted as a weighted $H^{-1}$-gradient flow (with weight $-1 / \partial_{n} S$ ) for the energy functional $E$ defined in (4.6).

Gradient flows in $H^{-1}$ are usually called surface diffusion flows; such flows arise in various materials science applications (cf. $[5,9,12,21])$. The energy in such applications always contains a surface energy term, whose shape gradient includes a mean curvature term. Consequently, the well-known surface diffusion flows include a surface Laplacian of curvature and therefore correspond to fourth-order parabolic differential equations. In the case of the limiting model (3.11), the energy is composed of bulk terms only, so that the resulting surface diffusion flow is only of second order. Consequently it makes sense to ask for a comparison principle at least if $S$ is fixed, i.e., the surface diffusion appears along a given potential. We shall see below that such a comparison principle indeed holds for the geometric flow, which seems slightly surprising for a surface diffusion model.

4.2. Stationary solutions and their stability. We start by showing that the energy is dissipated during the evolution. We can use again the form of the shape derivative to obtain

$$
\frac{d}{d t} \hat{E}[\bar{\Omega}(t)]=-\int_{\Gamma(t)} S V_{n} d \sigma=-\int_{\Gamma(t)} S \nabla_{\sigma} \cdot\left(\frac{\nabla_{\sigma} S}{\partial_{n} S}\right) d \sigma=\int_{\Gamma(t)} \frac{\left|\nabla_{\sigma} S\right|^{2}}{\partial_{n} S} d \sigma \leq 0,
$$

since $\partial_{n} S<0$ due to condition (3.4). Moreover, the equality sign in (4.11) only holds if $\nabla_{\sigma} S=0$; hence a stationary state can only be reached if $S$ is constant along the connected components of the interface. Note that this property also characterizes 
stationary points of the energy functional. If $S$ is constant along $\Gamma_{i}[W]=\{X(\sigma)+$ $\left.n(\sigma) V[W](\sigma): \sigma \in P_{i}\right\}$, the right hand side of (4.9) vanishes, since the integrals $\int(1+$ $\kappa V[W]) V^{\prime}[W](\hat{W}) d \sigma_{i}$ are the first variations of $\int\left(V[W]+\kappa V[W]^{2} / 2\right) d \sigma_{i}$ which have been chosen to be zero for all $W$.

Since the steady state solutions are exactly the stationary points of the energy functional, their stability can be studied via the second variation

$$
E^{\prime \prime}[0](W, \hat{W})=\lim _{\varepsilon \rightarrow 0} \frac{1}{\varepsilon}\left(E^{\prime}[\varepsilon W](\hat{W})-E^{\prime}[0](\hat{W})\right)
$$

of the energy functional. We assume a steady state $\bar{\Omega}[0]$ (with $S[0]$ constant along $\Gamma[0])$, and we shall need the expansions

$$
S[\varepsilon W](X+n V[\varepsilon W])=S[0](X)+\varepsilon\left(S^{\prime}[0](W)(X)+\partial_{n} S[0](X) W\right)+O\left(\varepsilon^{2}\right)
$$

and

$$
V^{\prime}[\varepsilon W](\hat{W})=\hat{W}-\varepsilon W_{0} \int \kappa W \hat{W} d \sigma
$$

leading to

$$
(1+\kappa V[\varepsilon W]) V^{\prime}[\varepsilon W](\hat{W})=\hat{W}+\varepsilon\left(\kappa W \hat{W}-W_{0} \int \kappa W \hat{W} d \sigma\right)+O\left(\varepsilon^{2}\right) .
$$

Using these in (4.9), we obtain

$$
E^{\prime \prime}[0](W, \hat{W})=-\int_{\Gamma[0]}\left(S^{\prime}(W)+\partial_{n} S W\right) \hat{W} d \sigma .
$$

In order to understand the stability of stationary surfaces, we inspect $E^{\prime \prime}[0](W, W)$. By using $\varphi=S^{\prime}(W)$ as a test function in the variational equation for $S^{\prime}$ we obtain that

$$
\int_{\Gamma[0]} S^{\prime}(W) W d \sigma=\int_{\Omega}\left(\left|\nabla S^{\prime}(W)\right|^{2}+\left(S^{\prime}(W)\right)^{2}\right) d x
$$

Note that the right-hand side can be interpreted as the square of an equivalent norm in $H^{-1 / 2}(\Gamma[0])$, and in particular the norm

$$
\|W\|_{H^{-1 / 2}}:=\sqrt{\int_{\Omega}\left(\left|\nabla S^{\prime}(W)\right|^{2}+\left(S^{\prime}(W)\right)^{2}\right) d x}
$$

is weaker than the $L^{2}$-norm. Hence, the quadratic form corresponding to the second variation can be written as the sum of a positive and a negative term:

LEMMA 4.2. The second variation of the energy functional defined in (4.6) is given by

$$
E^{\prime \prime}[0](W, W)=-\int_{\Gamma[0]} \partial_{n} S W^{2} d \sigma-\|W\|_{H^{-1 / 2}}^{2}
$$

with the norm $\|\cdot\|_{H^{-1 / 2}}$ defined above. 
Since the positive term is a weighted $L^{2}$-norm, the interface is stable with respect to high-frequency perturbations and possibly unstable with respect to low-frequency perturbations. This supports our assumption above that the dynamics has a regularizing effect on the interface. Examples of both stable and unstable steady states will be presented in the following section.

We finally mention that the same stability result can be derived equivalently (but with more involved arguments on constrained shape optimization) by incorporating the volume constraints via a Lagrange functional. We demonstrate this procedure for the simplest case of a connected free boundary of one connected plateau $\bar{\Omega}$ with fixed volume $m$. The Lagrangian is then given by

$$
L[\bar{\Omega} ; \lambda]:=\hat{E}[\bar{\Omega}]+\lambda\left(\int_{\bar{\Omega}} d x-m\right),
$$

where $\lambda \in \mathbb{R}$ is a Lagrange parameter to be determined. By general results on shape variations (cf. [10]) one obtains (with $\Gamma=\partial \bar{\Omega} \backslash \partial \Omega$ ) for a perturbation with normal velocity $V_{n}$

$$
L^{\prime}[\bar{\Omega} ; \lambda] V_{n}=-\int_{\Gamma} S[\bar{\Omega}] V_{n} d \sigma+\lambda \int_{\Gamma} V_{n} d \sigma
$$

Stationary solutions are characterized by $L^{\prime}[\bar{\Omega} ; \lambda] V_{n}=0$ for all possible velocities $V_{n}$, i.e., $S[\bar{\Omega}]=\lambda$ on $\Gamma$. Stability is equivalently obtained by the positivity of the second variation (for normal velocities with constant extension in normal direction away from $\Gamma)$, which is given by

$$
L^{\prime \prime}[\bar{\Omega} ; \lambda]\left(V_{n}, V_{n}\right)=-\int_{\Gamma}\left(\partial_{n} S[\bar{\Omega}]+S[\bar{\Omega}] \kappa\right) V_{n}^{2} d \sigma-\left\|V_{n}\right\|_{H^{-1 / 2}}^{2}+\lambda \int_{\Gamma} \kappa V_{n}^{2} d \sigma,
$$

where $\kappa$ denotes the mean curvature of the interface $\Gamma$. One observes that for stationary shapes the curvature terms cancel, and the stability of $\bar{\Omega}$ with respect to perturbations $V_{n}$ again reduces to the condition

$$
\int_{\Gamma} \partial_{n} S[\bar{\Omega}] V_{n}^{2} d \sigma+\left\|V_{n}\right\|_{H^{-1 / 2}}^{2}<0
$$

4.3. Linear stability of quasi-1D surfaces. From the asymptotic interface motion (3.11) obtained above we see that the plateaus move differently in multiple dimensions than in the one-dimensional case analyzed with exponential asymptotics in [11]. If the interface has a quasi-1D shape, i.e., if $\Omega$ and $\bar{\Omega}(0)$ are rectangles with common facets, then the multidimensional problem can still be formulated as an equivalent one-dimensional problem using symmetry reductions. It is an interesting questions how small symmetry-breaking perturbations of such a quasi-1D surface behave, i.e., whether the quasi-1D shape is stable or not. In order to gain insight into this issue, we consider the stability of the stationary shape

$$
\hat{\Omega}:=\left\{\left(x_{1}, x_{2}\right) \in \Omega \mid 0<x_{2}<a\right\}, \quad \text { with } \Omega=(0,1) \times(0, b) \text { and } a \in(0, b),
$$

under normal perturbations $V[W]$ with $W=W\left(x_{1}\right)$, such that $\int_{0}^{1} W\left(x_{1}\right) d x_{1}=0$. These perturbations can be decomposed into the Fourier modes $W_{k}=\cos \left(k \pi x_{1}\right)$ with positive integers $k$. Obviously, it is sufficient to investigate their stability. 
In order to calculate the second variation of the energy (4.12), we must first determine $S$ and $S^{\prime}$. Thanks to (4.3), $S$ is the weak solution of

$$
\int_{0}^{1} \int_{0}^{b}(\nabla S \cdot \nabla \varphi+S \varphi) d x_{2} d x_{1}=\int_{0}^{1} \int_{0}^{a} \varphi\left(x_{1}, x_{2}\right) d x_{2} d x_{1} \quad \forall \varphi \in H^{1}(\Omega) .
$$

Due to the symmetries of the problem we can look for a solution $S=S\left(x_{2}\right)$, which satisfies

$$
-\frac{d^{2} S}{d x_{2}^{2}}+S=\left\{\begin{array}{l}
1 \text { if } x_{2}<a \\
0 \text { else }
\end{array}\right.
$$

subject to $\frac{d S}{d x_{2}}=0$ at $x_{2}=0, b$. Hence, the equation can be solved to obtain

$$
S\left(x_{2}\right)= \begin{cases}1+\alpha \cosh x_{2} & \text { if } x_{2}<a \\ \beta \cosh \left(b-x_{2}\right) & \text { else. }\end{cases}
$$

for constants $\alpha, \beta$ to be determined from the continuity of $S$ and its first derivative at $x_{2}=a$, implying

$$
1+\alpha \cosh a=\beta \cosh (b-a), \quad \alpha \sinh a=-\beta \sinh (b-a)
$$

and hence,

$$
\beta=\frac{\sinh a}{\cosh (b-a) \sinh a+\sinh (b-a) \cosh a}, \quad \alpha=-\frac{\beta \sinh (b-a)}{\sinh a} .
$$

On the other hand, it follows from (4.8) that $S^{\prime}$ solves

$$
\int_{0}^{1} \int_{0}^{b}\left(\nabla S^{\prime} \cdot \nabla \varphi+S^{\prime} \varphi\right) d x_{2} d x_{1}=\int_{0}^{1} W \varphi\left(a, x_{1}\right) d x_{1} \quad \forall \varphi \in H^{1}(\Omega) .
$$

This equation is equivalent to

$$
-\Delta S^{\prime}+S^{\prime}=0 \quad \text { for } x_{2} \in(0, a) \cup(a, b),
$$

with $\frac{\partial S^{\prime}}{\partial n}=0$ on $\partial \Omega$ and the interface condition

$$
\frac{\partial S^{\prime}}{\partial x_{2}}\left(x_{1}, a_{-}\right)-\frac{\partial S^{\prime}}{\partial x_{2}}\left(x_{1}, a_{+}\right)=W .
$$

This problem can be solved by separation of variables. Inserting $W=\cos \left(k \pi x_{1}\right)$, we can compute $S^{\prime}\left(x_{1}, x_{2}\right)=u\left(x_{2}\right) \cos k \pi x_{1}$, with $u$ satisfying

$$
-\frac{\partial^{2} u}{\partial x_{2}^{2}}+\left(1+k^{2} \pi^{2}\right) u=0 \quad \text { for } x_{2} \in(0, a) \cup(a, b) .
$$

To simplify notations, set $\vartheta_{k}:=\sqrt{1+k^{2} \pi^{2}}$; then

$$
u\left(x_{2}\right)= \begin{cases}\gamma \cosh \left(\vartheta_{k} x_{2}\right) & \text { if } x_{2}<a \\ \eta \cosh \left(\vartheta_{k}\left(b-x_{2}\right)\right) & \text { else. }\end{cases}
$$

The coefficients $\gamma$ and $\eta$ can be obtained from the interface conditions as

$$
\gamma \cosh \left(\vartheta_{k} a\right)=\eta \cosh \left(\vartheta_{k}(b-a)\right) \quad \vartheta_{k} \gamma \sinh \left(\vartheta_{k} a\right)+\eta \vartheta_{k} \sinh \left(\vartheta_{k}(b-a)\right)=1
$$


which can be solved to obtain

$$
\gamma=\frac{1}{\vartheta_{k}} \frac{\cosh \left(\vartheta_{k}(b-a)\right)}{\sinh \left(\vartheta_{k} b\right)}, \quad \eta=\frac{1}{\vartheta_{k}} \frac{\cosh \left(\vartheta_{k} a\right)}{\sinh \left(\vartheta_{k} b\right)} .
$$

Now we are ready to calculate the terms in (4.12). Since $\|W\|_{H^{-1 / 2}}^{2}=$ $\int_{0}^{1} S^{\prime}\left(x_{1}, a\right) W d x_{1}$ and $\partial_{n} S=-\beta \sinh (b-a)$ on $\partial \hat{\Omega}$, we have that

$$
\begin{aligned}
\hat{E}^{\prime \prime}(\hat{\Omega})(W, W) & =(\beta \sinh (b-a)-u(a)) \int_{0}^{1} \cos ^{2}\left(k \pi x_{1}\right) d x_{1} \\
& =\frac{1}{2 k \pi}(\beta \sinh (b-a)-u(a))(\cos (k \pi) \sin (k \pi)+k \pi) .
\end{aligned}
$$

LEMma 4.3. The quasi-one dimensional solution (4.13) of the interface motion (3.11) is linearized stable if, for all $k \geq 1$,

$$
\frac{\sinh a \sinh (b-a)}{\cosh (b-a) \sinh a+\sinh (b-a) \cosh a}>\frac{\cosh \left(\vartheta_{k} a\right) \cosh \left(\vartheta_{k}(b-a)\right)}{\vartheta_{k} \sinh \left(\vartheta_{k} b\right)} .
$$

Note that the formula (4.15) stays the same if one exchanges $a$ and $(b-a)$, implying that quasi one-dimensional plateaus and holes will behave the same way. This property reflects the fact that the original equations (1.1) and (1.2) are invariant under the change of variables $\varrho \rightarrow(1-\varrho)$ and $S \rightarrow(1-S)$.

In order to understand the stability of these quasi-1D solutions qualitatively, it is instructive to study some limiting cases of (4.15):

For $k \rightarrow \infty$ we obtain that $\frac{\cosh \left(\vartheta_{k} a\right) \cosh \left(\vartheta_{k}(b-a)\right)}{\sinh \left(\vartheta_{k} b\right)} \rightarrow \frac{1}{2}$. Thus, the right hand side of (4.15) converges to zero for $k$ sufficiently large, and therefore $\hat{E}^{\prime \prime}(\hat{\Omega})(W, W)>0$ for all choices of $a$ and $b$. Hence, the quasi one-dimensional state is stable with respect to high frequency perturbations, as we could already infer directly from (4.12).

For fixed $k=\bar{k}$, the stability of the solutions depends on the parameters. If we let $a \rightarrow 0$, the left hand side of (4.15) converges to zero, whereas the right hand side converges to $\vartheta_{\bar{k}}^{-1} \operatorname{coth}\left(\vartheta_{\bar{k}} b\right)$. Keeping also $b$ fixed, we can consequently always choose an $a$ small enough such that (4.15) does not hold and the solution becomes unstable. In particular, the same then applies to all perturbations with a lower frequency than the chosen one, i.e., for all $\vartheta_{k}<\vartheta_{\bar{k}}$. Due to the above mentioned symmetry in $a$ and $(b-a)$ of (4.15), the same argument applies if we let $a \rightarrow b$.

To see what happens if $a$ is neither very small nor large compared to $b$, we set $a=b / 2$, yielding

$$
\vartheta_{k} \tanh \left(\frac{b}{2}\right) \tanh \left(\frac{\vartheta_{k} b}{2}\right)>1 .
$$

Since $\vartheta_{k}>1$, this inequality holds for all $\vartheta_{k}$ if $b$ is large enough. On the other hand, (4.16) is violated in the limit $b \rightarrow 0$. Consequently, solutions loose their stability if $b$ is below a minimal value.

4.4. Level set formulation and comparison principle. In order to gain some insight into the basic properties of the interface motion, we consider the motion in a given potential $S$, i.e.,

$$
V_{n}=\nabla_{\sigma} \cdot\left(\frac{1}{\partial_{n} S} \nabla_{\sigma} S\right) \quad \text { on } \Gamma(t) \times(0, T) .
$$


From the derivation of the model it is not obvious whether there is a comparison principle between two interfaces, i.e., if the property $\bar{\Omega}(t) \subset \overline{\hat{\Omega}}(t)$ is conserved in time. The study of a comparison principle can be performed in a nice way via a level set approach (cf. $[23,22]$ ), i.e., we look for a function $\varphi: \Omega \times[0, T] \rightarrow \mathbb{R}$, such that

$$
\bar{\Omega}(t)=\{x \in \Omega \mid \varphi(x, t)<0\}, \quad \Gamma(t)=\{x \in \Omega \mid \varphi(x, t)=0\} .
$$

It is well-known that the normal and normal velocity in terms of the level set function are given by

$$
n=\frac{\nabla \varphi}{|\nabla \varphi|}, \quad V_{n}=-\frac{\partial_{t} \varphi}{|\nabla \varphi|}
$$

The differential operator on the surface can be rewritten as (cf. [3])

$$
\nabla_{\sigma} \cdot\left(\frac{1}{\partial_{n} S} \nabla_{\sigma} S\right)=\frac{1}{|\nabla \varphi|} \nabla \cdot\left(\frac{|\nabla \varphi|}{\partial_{n} S} \mathbf{P} \nabla S\right), \quad \mathbf{P}=\mathbf{I}-\frac{\nabla \varphi}{|\nabla \varphi|} \otimes \frac{\nabla \varphi}{|\nabla \varphi|} .
$$

Hence,

$$
\partial_{t} \varphi=-\nabla \cdot\left(\frac{|\nabla \varphi|^{2}}{\nabla \varphi \cdot \nabla S} \mathbf{P} \nabla S\right)=-\nabla \cdot\left(\frac{|\nabla \varphi|^{2}}{\nabla \varphi \cdot \nabla S} \nabla S\right)+\nabla \cdot(\nabla \varphi),
$$

so that we end up with a fully nonlinear second-order parabolic evolution equation, which can be analyzed using the theory of viscosity solutions for equations of the form (cf. [7])

$$
\partial_{t} \varphi=F\left(x, t, \nabla \varphi, D^{2} \varphi\right) .
$$

The main condition needed for the analysis (besides regularity) is monotonicity of the right-hand side $F$ with respect to the Hessian $D^{2} \varphi$. Note that in the case of (4.18)

$$
F(x, t, p, \mathbf{A})=\operatorname{tr}(\mathbf{A})-\frac{2 p \cdot(\mathbf{A} \nabla S(x, t))}{p \cdot \nabla S(x, t)}+\frac{|p|^{2}(\nabla S(x, t)) \cdot(\mathbf{A} \nabla S(x, t))}{(p \cdot \nabla S(x, t))^{2}}+G(x, t, p) .
$$

Hence, with the notation $q=\nabla S(x, t)$ we have for $\mathbf{A} \geq \mathbf{B}$

$$
\begin{aligned}
F(x, t, p, \mathbf{A})-F(x, t, p, \mathbf{B}) & =\operatorname{tr}(\mathbf{A}-\mathbf{B})-\frac{2 p \cdot((\mathbf{A}-\mathbf{B}) q)}{p \cdot q}+\frac{|p|^{2} q \cdot((\mathbf{A}-\mathbf{B}) q)}{(p \cdot q)^{2}} \\
& \geq \operatorname{tr}(\mathbf{A}-\mathbf{B})-\frac{p \cdot((\mathbf{A}-\mathbf{B}) p)}{|p|^{2}} \\
& \geq \sum_{j=1}^{d} \lambda_{j}-\lambda_{d} \geq \sum_{j=1}^{d-1} \lambda_{j} \geq 0
\end{aligned}
$$

where $0 \leq \lambda_{1} \leq \cdots \leq \lambda_{d}$ are the eigenvalues of the positive semidefinite matrix $\mathbf{A}-\mathbf{B}$.

The monotone dependence of $F$ implies that for solutions $\varphi$ of (4.18) satisfying the entropy condition $\nabla \varphi \cdot \nabla S<0$ a comparison principle holds. Now let $\varphi$ be the solution of (4.18) with zero level set $\Gamma(t)$ and $\hat{\varphi}$ be the solution with zero level set $\hat{\Gamma}(t)$. If $\bar{\Omega}_{0} \subset \bar{\Omega}_{0}$, this means that we can choose initial level set functions with $\varphi(., 0) \geq \hat{\varphi}(., 0)$. Then by the comparison principle for level set equation we obtain that $\varphi(., t) \geq \hat{\varphi}(., t)$ for all $t \geq 0$, and thus

$$
\bar{\Omega}_{0} \subset \overline{\hat{\Omega}}_{0} \quad \Rightarrow \quad \bar{\Omega}(t) \subset \overline{\hat{\Omega}}(t), \quad \forall t \geq 0 .
$$


The comparison principle (4.19) seems to be the first known for a surface diffusion model; it is obviously not true for the previously studied fourth-order models. In the general case of a coupling with $S$ via an elliptic differential equation, the comparison principle will be violated due to the nonlocal interaction, but for a small time step one could always think of approximating the geometric motion with given $S$, so that one expects a rather well-behaved geometric motion without effects like pinch-off (which is also found in numerical experiments).

\section{Numerical experiments}

In the following we present several numerical experiments illustrating the asymptotic behavior of the Keller-Segel model with small diffusivity, and the theoretical results found above.

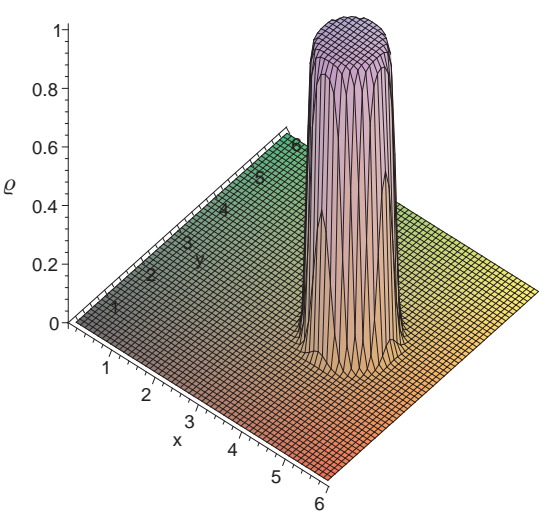

(a) Density $\varrho$

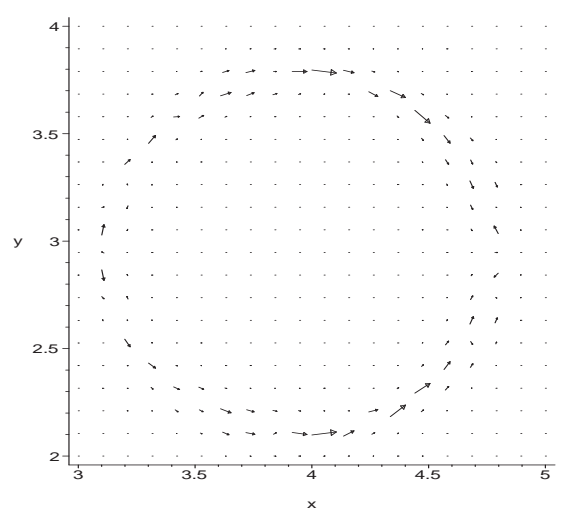

(b) Density flux $\varrho(1-\varrho) \nabla S-\varepsilon \nabla \varrho$

FIG. 5.1. Numerical solution of (1.1)-(1.4) showing a plateau moving in positive $x$-direction and the corresponding density flux.

5.1. Formation of plateaus and coarsening. The formation of plateaus from a random initial distribution of cells is illustrated in Figure 3.1, where system (1.1)-(1.4) was solved numerically using a finite difference scheme. The computational domain is $\Omega=(0,2)^{2}$, the diffusivity $\varepsilon=10^{-4}$. As initial value we choose pointwise independent uniformly distributed random variables in $[0.1,0.11]$, i.e., $\varrho_{I}$ can be interpreted as a small random perturbation of the constant density 0.105 . As predicted by the asymptotic analysis, plateaus form after some time (see (a)-(d)), and then a coarsening of the plateaus starts (see change from (d) to (e)).

5.2. Surface diffusion. Figure 5.1 shows a high density-region of $\varrho$ on the domain $\Omega=(0,6)^{2}$ moving in the positive $x$-direction and the corresponding density flux $\varrho(1-\varrho) \nabla S-\varepsilon \nabla \varrho$. We can see that inside the plateau, the flux is practically zero. The biological interpretation of this observation is that due to the dense packing of cells in the interior regions of the plateau, cell movement can only occur along the plateau boundary. This type of behavior is actually observed around aggregates of Dictyostelium discoideum.

5.3. Stability of quasi-1D surfaces. We investigate the stability of quasi-1D surfaces numerically by choosing quasi-one dimensional initial conditions according 


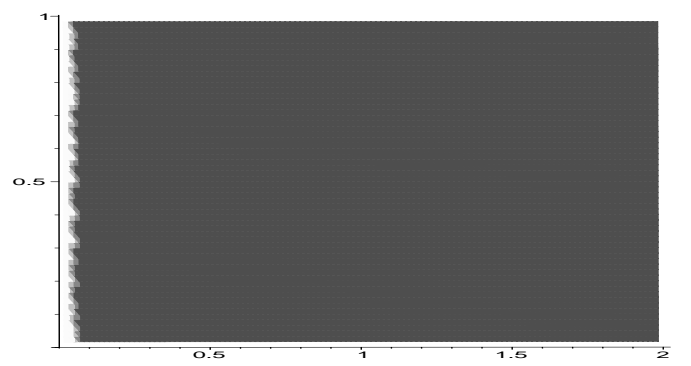

(a) $t=0$

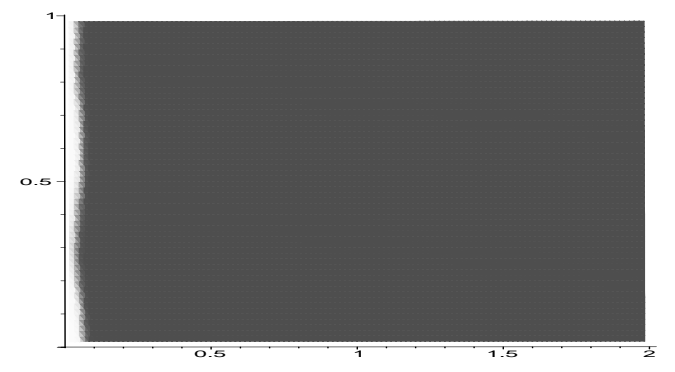

(b) $t=0.01$

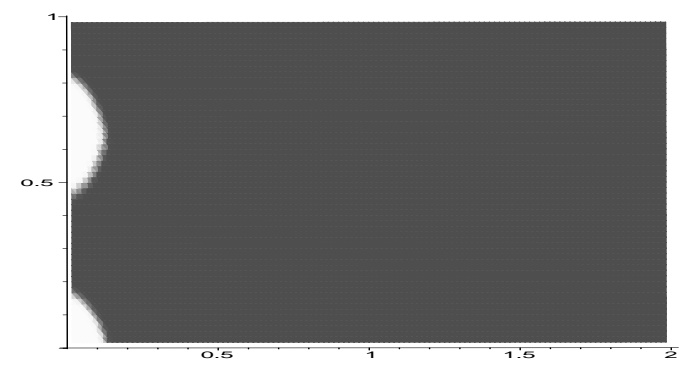

(c) $t=0.03$

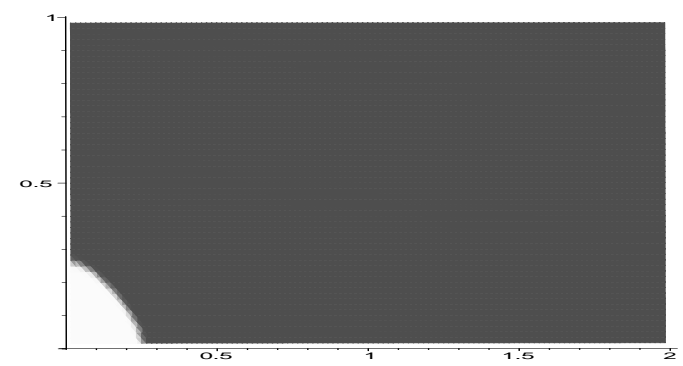

(d) $t=0.28$

FIG. 5.2. Numerical solution of (1.1)-(1.4) with $\varepsilon=10^{-4}$ and the quasi-one dimensional initial condition (5.1) with $a=0.05$. Regions with a high density of $\varrho$ are colored white, low density regions dark grey. Note that all plots in this section have been rotated by 90 degrees.

to Subsection 4.3, i.e.,

$$
\varrho_{I}\left(x_{1}, x_{2}\right)=\left\{\begin{array}{l}
1 \text { if } x_{2}<a \pm \text { random perturbation } \\
0 \text { else }
\end{array}\right.
$$




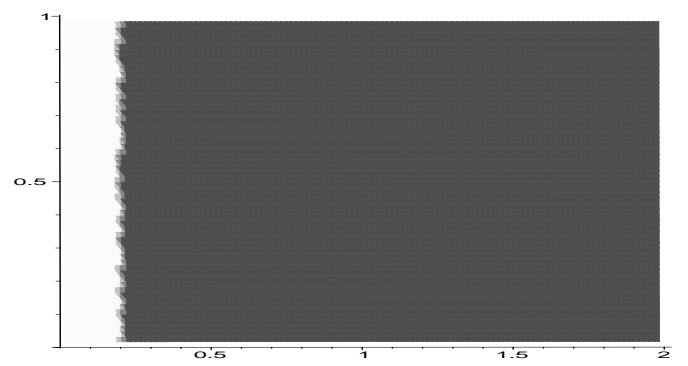

(a) $t=0$

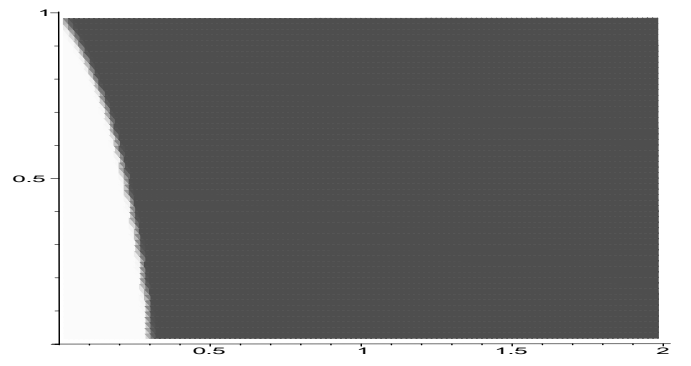

(b) $t=0.22$

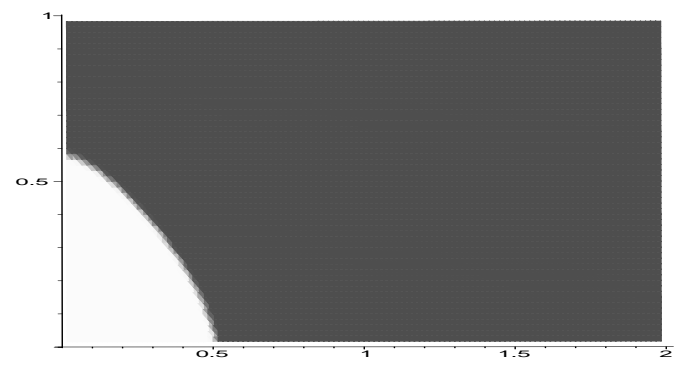

(c) $t=0.44$

FIG. 5.3. Numerical solution of (1.1)-(1.4) with parameters and initial condition as above and $a=0.2$.

where the amplitude of the random perturbation equals the grid size.

The results in Section 4.3 imply that while letting $a \rightarrow 0$ and keeping $b$ fixed, the stability condition (4.15) is violated for larger and larger wavenumbers $k$. Hence, if we add a small, random perturbation to two sets of initial data with the same value for $b$ but different values for $a$, we expect the evolution of higher frequency patterns for the initial data with the smaller $a$, as illustrated in the following two figures:

Figure 5.2 shows the cell density $\varrho$ in the domain $\Omega=(0,1) \times(0,2)$ for different points in time. Since we describe phenomena that occur on the diffusive time scale, the time given in the figure captions in this and the following sections is already rescaled accordingly. We can see how a pattern starts to grow, evolving into two plateaus at the domain boundary. Eventually, these plateaus merge and the stationary state of a single plateau in the corner is approached.

For Figure 5.3, we chose similar initial conditions, but a larger parameter $a$ than in the last figure. Now, a pattern of lower frequency evolves, gradually forming one 


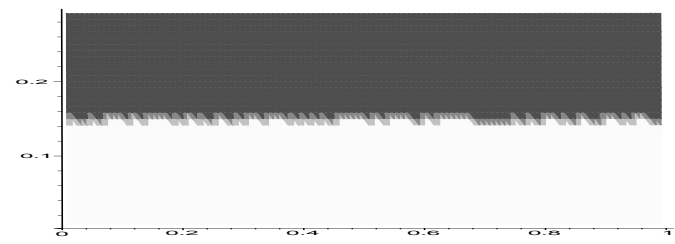

(a) $t=0$

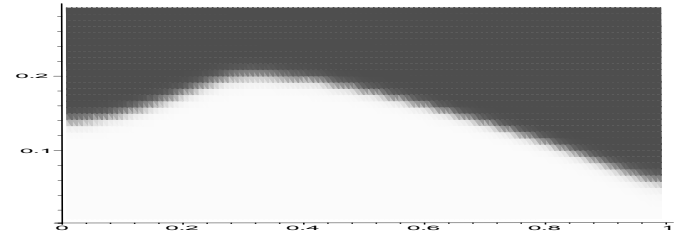

(b) $t=0.05$

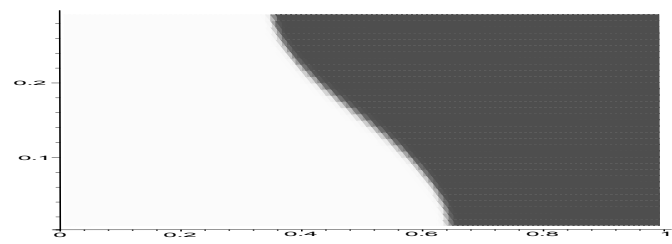

(c) $t=0.08$

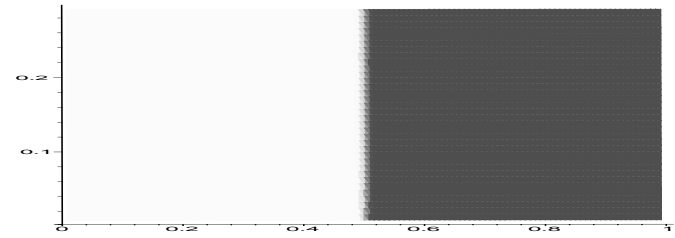

(d) $t=0.4$

FIG. 5.4. Numerical solution of (1.1)-(1.4) on $\Omega=(0,1) \times(0,0.3)$ with parameters and initial condition as above and $a=0.05$.

single plateau in the corner.

Finally, Figure 5.4 illustrates the instability of solutions with $a=b / 2$ if $b$ does not fulfill (4.16).

5.4. Long-time behavior on a rectangular domain. Our first example of long-time behavior is carried out on the rectangular domain $\Omega=(0,4) \times(0,1)$. Figure 5.5 shows the cell density at two different points in time, as well as isolines of the corresponding chemical concentration $S$. Starting from initial conditions consisting of a small strip of mass in the left and a larger strip in the right corner, the cell density evolves into a planar plateau on the right and a small plateau in the left corner. Whereas the value of the chemical density along the boundary of the large plateau is already practically constant in the first picture 5.5(a), the smaller plateau approaches its stationary shape much later, see 5.5(b).

In Figure 5.6, we start with random initial conditions $\varrho_{I}(x) \in[0.7,0.71]$ and compute the evolution of the cell density on the long but narrow rectangular domain $\Omega=(0,2) \times(0,0.4)$. This simulation turns out to show an especially interesting dy- 


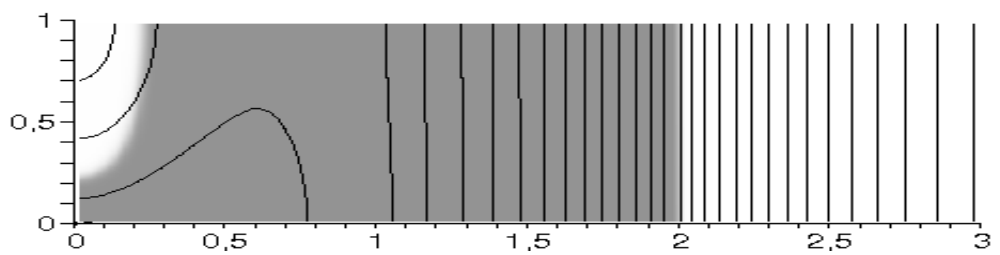

(a) $t=0.22$

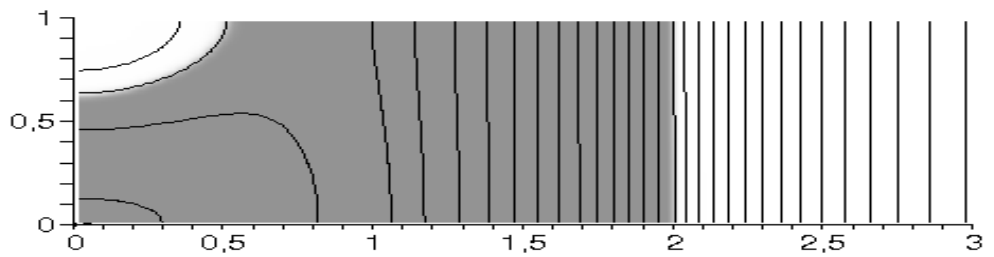

(b) $t=11$

FIG. 5.5. Numerical solution of (1.1)-(1.4) with $\varepsilon=10^{-3}$ and initial conditions consisting of a small strip of mass in the left and a larger strip in the right corner. Regions with a high density of $\varrho$ are colored white, low density regions are grey. Black lines are isolines of the chemical concentration $S$.

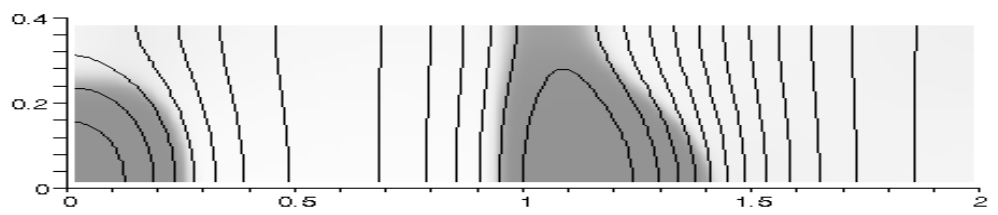

(a) $t=0.02$

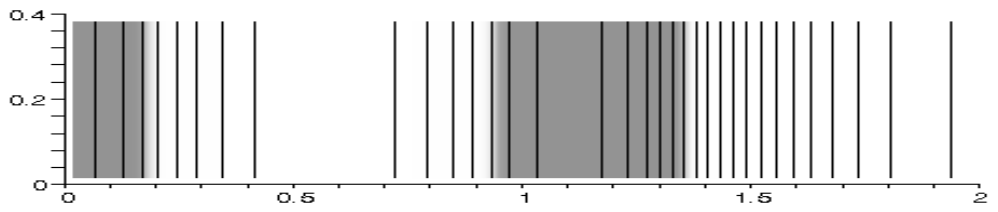

(b) $t=110$

FIG. 5.6. Numerical solution of (1.1)-(1.4) with $\varepsilon=5 \times 10^{-4}$ and random initial conditions $\varrho_{I}(x) \in[0.7,0.71]$. Black lines are isolines of the chemical concentration $S$.

namical behavior: after a fast transition from the initial data to a solution with two plateaus in 5.6(a), the cell density assumes a quasi-one dimensional shape in 5.6(b), instead of converging to stationary shapes in the corner like above. Although the chemical $S$ is now practically constant along the boundary, and thus the stationary state of the dynamics described by equation (3.11) has almost been reached, we know from the analysis of the one-dimensional problem that this is not a stationary state of the full system. Hence, we expect that for this set of initial conditions, dynamics cannot be only described on the fast hyperbolic and the slow diffusive time scale, but that there is a third, exponentially slow time scale as described in [11] for the one-dimensional system. Consequently, exponentially slow movement of the plateaus towards each other or the domain boundary can be anticipated. 


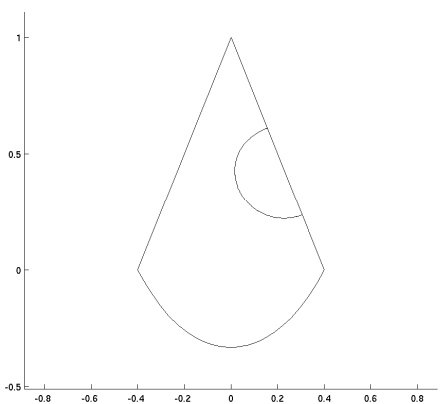

(a) Level set $\{\varrho(., t)=0.5\}, t=0.01$

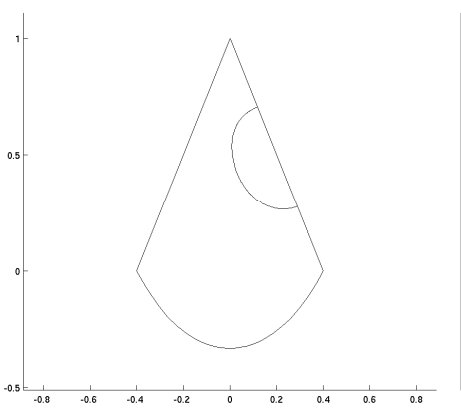

(c) Level set $\{\varrho(., t)=0.5\}, t=0.04$

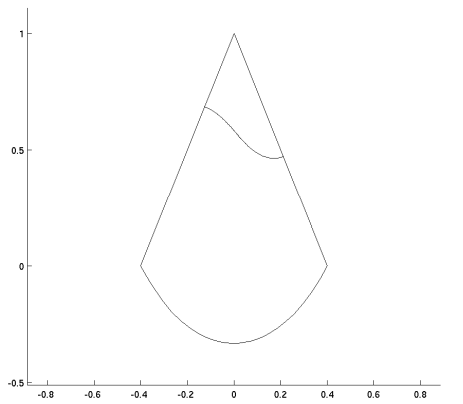

(e) Level set $\{\varrho(., t)=0.5\}, t=0.08$

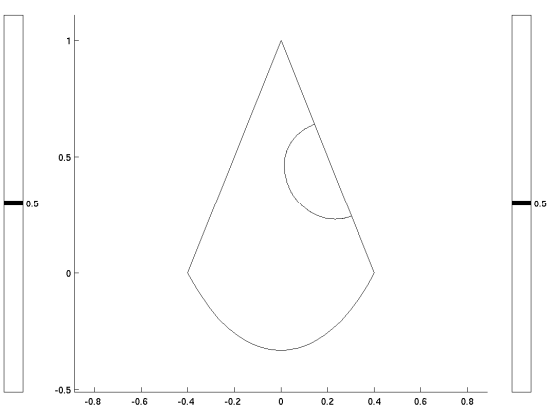

(b) Level set $\{\varrho(., t)=0.5\}, t=0.02$

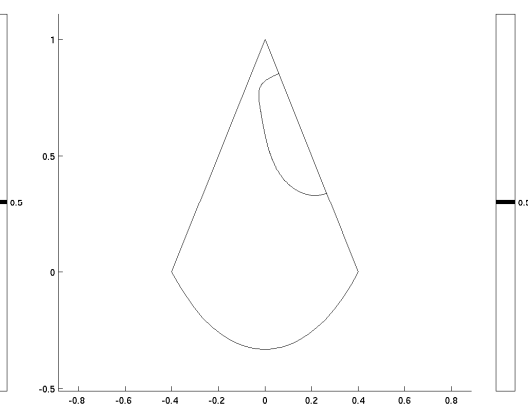

(d) Level set $\{\varrho(., t)=0.5\}, t=0.06$

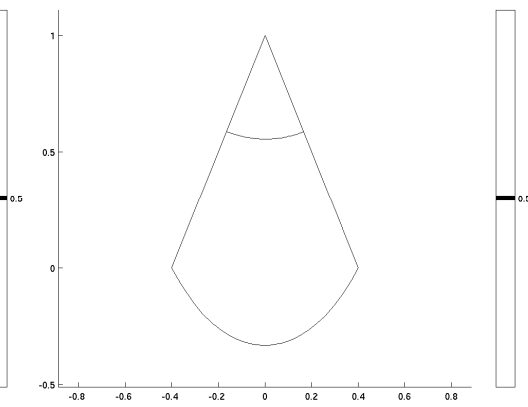

(f) Level set $\{\varrho(., t)=0.5\}, t=0.16$

FIG. 5.7. Numerical simulation of a plateau moving to the corner of a cone. The shape displays the level set $\{\varrho(., t)=0.5\}$.

5.5. Long-time behavior on a $2 \mathrm{D}$ cone. In order to illustrate the effect of the geometry of $\Omega$ on the long-time dynamics, we display the results of a simulation on a cone. The following example is solved numerically using a finite element method and semi-implicit time stepping. The initial value is

$$
\varrho_{I}(x, y)=e^{-\left(25(x-0.2)^{2}+(y-0.4)^{2}\right)},
$$

which corresponds to a peak close to $(0.2,0.4)$. The diffusion coefficient is $2 \times 10^{-3}$ and the time step used for the simulation is $2.5 \times 10^{-2}$. We illustrate the dynamics of the interface, i.e., the level set $\left\{\varrho=\frac{1}{2}\right\}$, in Figure 5.7. The interface has nonempty intersection with $\partial \Omega$ for all times in this case, and moves into the corner of the cone. From (f) one observes more clearly the symmetry of the stationary shape. 


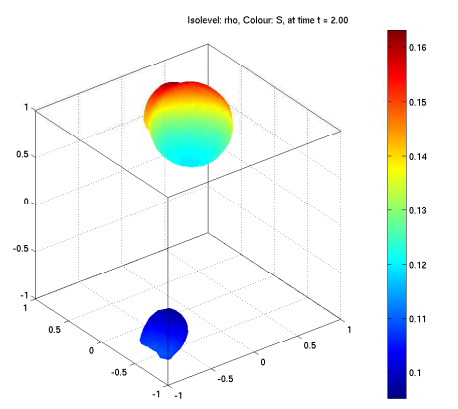

(a) Level set $\{\varrho(., t)=0.5\}, t=0.01$

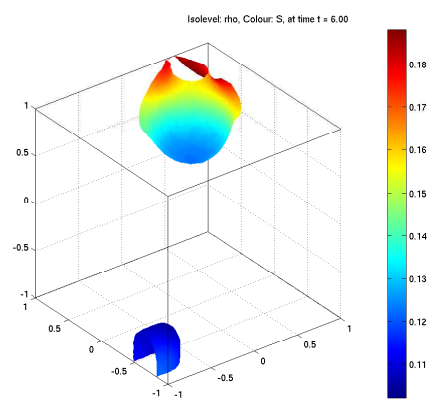

(c) Level set $\{\varrho(., t)=0.5\}, t=0.03$

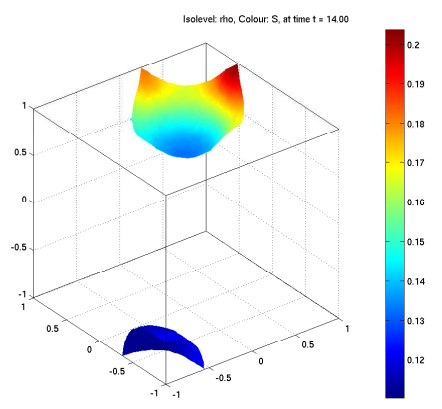

(e) Level set $\{\varrho(., t)=0.5\}, t=0.07$

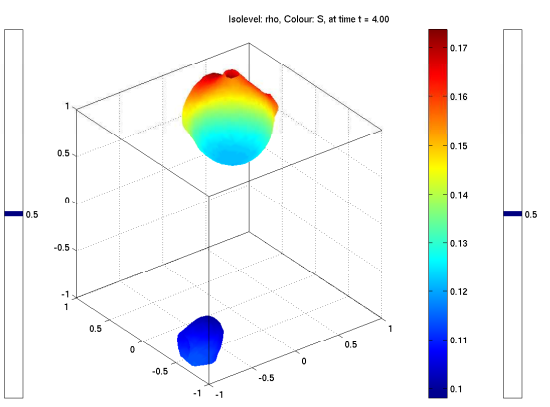

(b) Level set $\{\varrho(., t)=0.5\}, t=0.02$

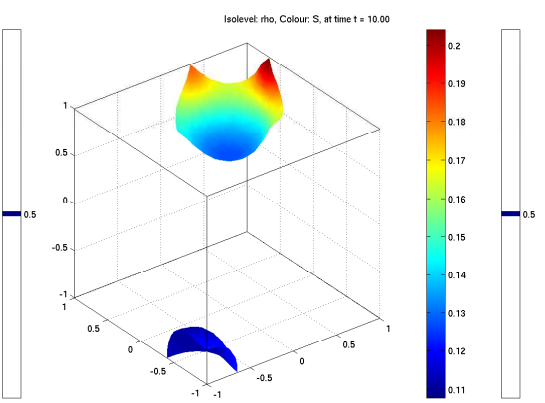

(d) Level set $\{\varrho(., t)=0.5\}, t=0.05$

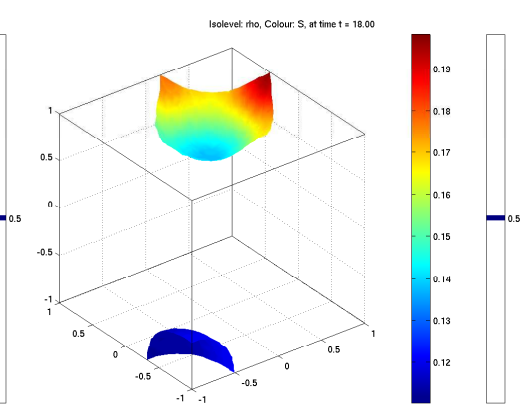

(f) Level set $\{\varrho(., t)=0.5\}, t=0.06$

FIG. 5.8. Numerical simulation of three-dimensional plateaus on a cube. The shape displays the level set $\{\varrho(., t)=0.5\}$.

5.6. Long-time behavior in $3 \mathrm{D}$. Finally, we illustrate the results of threedimensional simulations of the long-time behavior. In this case, we choose the domain $\Omega=(-1,1)^{3}, \varepsilon=0.005$, and time step $\tau=0.02$. We start with two plateaus close to opposite corners of the cube, namely the initial value

$$
\varrho_{I}(x, y, z)=\left\{\begin{array}{l}
0.95 \text { if } \sqrt{(x-0.5)^{2}+(z-0.5)^{2}+(y-0.6)^{2}} \leq 0.4 \\
0.95 \text { if } \sqrt{(x+0.7)^{2}+(z+0.7)^{2}+(y+0.6)^{2}} \leq 0.25 \\
0.05 \text { else. }
\end{array}\right.
$$

Six different time steps of the evolution are illustrated in Figure 5.8 via contour plots of the level sets $\{\varrho(., t)=0.5\}$ (other level sets of $\varrho$ look very similar due to the plateau 


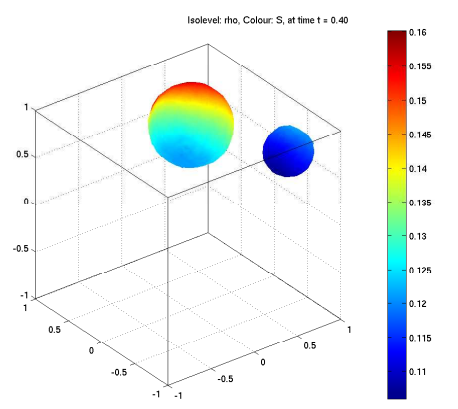

(a) Level set $\{\varrho(., t)=0.5\}, t=0.002$

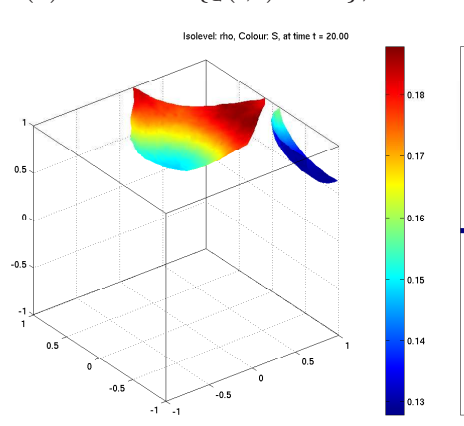

(c) Level set $\{\varrho(., t)=0.5\}, t=0.1$

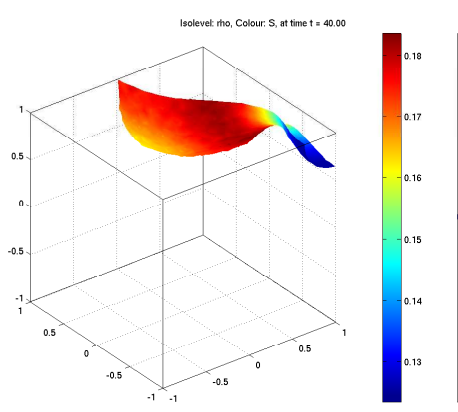

(e) Level set $\{\varrho(., t)=0.5\}, t=0.2$

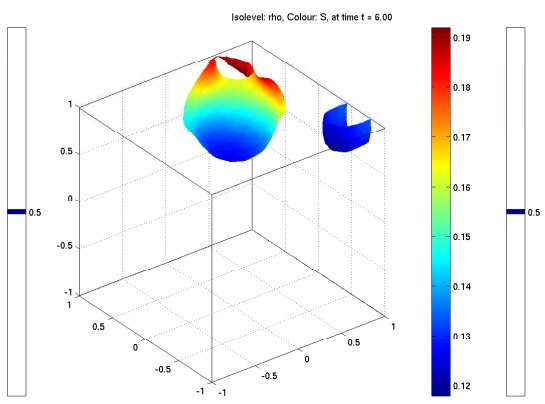

(b) Level set $\{\varrho(., t)=0.5\}, t=0.03$

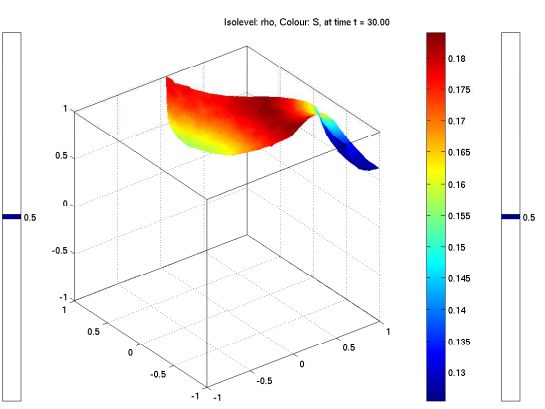

(d) Level set $\{\varrho(., t)=0.5\}, t=0.15$

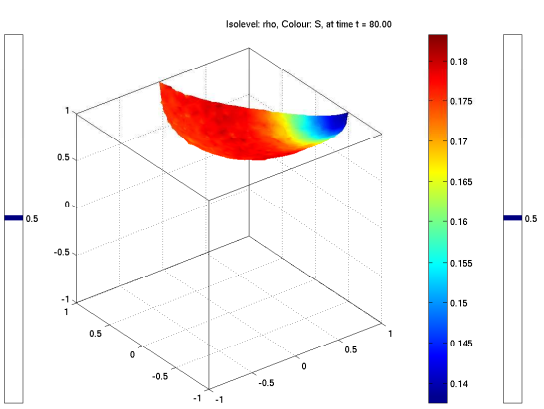

(f) Level set $\{\varrho(., t)=0.5\}, t=0.4$

FIG. 5.9. Numerical simulation of three-dimensional plateaus on a cube. The shape displays the level set $\{\varrho(., t)=0.5\}$.

structure of solutions). One observes that both plateaus move to the closest corner of the cube and the interfaces form local stationary shapes.

The behavior changes if the initial value consists of two plateaus in neighbouring corners of the cube: we choose

$$
\varrho_{I}(x, y, z)=\left\{\begin{array}{l}
0.95 \text { if } \sqrt{(x-0.5)^{2}+(z-0.5)^{2}+(y-0.6)^{2}} \leq 0.4 \\
0.95 \text { if } \sqrt{(x-0.7)^{2}+(z-0.7)^{2}+(y+0.6)^{2}} \leq 0.25 \\
0.05 \text { else. }
\end{array}\right.
$$

Again, the analogous contour plots as above are shown for six different time steps of the evolution in Figure 5.9. In this case one observes that the larger plateau grows at the expense of the smaller one, and finally the smaller one disappears before the 
interface tends to a different equilibrium shape than above.

Acknowledgments. The work of M.B. has been supported by the Austrian Science Foundation through project SFB F 013 / 08 and the Johann Radon Institute for Computational and Applied Sciences (Austrian Academy of Sciences). The work of Y.D.S. has been partially supported by the Johann Radon Institute and the WWTF, project no. MA39. The work of C.S. has been supported by the Austrian Science Foundation under grant nos. W008 and P16174-N05, and by the EU-Marie Curie Early Stage Training Network DEASE (contract no. MEST-CT-2005-021122).

\section{REFERENCES}

[1] G. Arfken and H. Weber, Mathematical Methods for Physicists, Academic Press, 1995.

[2] G. Barles, H.M. Soner and P.E. Souganidis, Front propagation and phase field theory, SIAM J. Control Optim., 31, 439-469, 1993.

[3] M. Burger, Elliptic partial differential equations on implicit curves and surfaces: variational formulation and finite element approximation, CAM-Report, submitted, UCLA, 05-46, 2005.

[4] M. Burger, M. DiFrancesco and Y. Dolak-Struß, The Keller-Segel model for chemotaxis with prevention of overcrowding: linear vs. nonlinear diffusion, SIAM J. Math. Anal., 38, 1288$1315,2006$.

[5] J.W. Cahn and J.E. Taylor, Surface motion by surface diffusion, Acta Metall. Mater., 42, 1045-1063, 1994.

[6] V. Calvez and Y. Dolak-Struß, Asymptotic behavior of a wwo-dimensional Keller-Segel model with and without density control, Proc. of the ECMTB conf., Dresden, 2005.

[7] M. Crandall, H. Ishii and P.L. Lions, User's guide to viscosity solutions of second order partial differential equations, Bull. Am. Math. Soc., 27, 1-67, 1992.

[8] B. Perthame and A. Dalibard, Existence of solutions of the hyperbolic Keller-Segel model, Trans. AMS, to appear, 2007.

[9] F. Davi and M.E. Gurtin, On the motion of a phase interface by surface diffusion, J. Appl. Math. Phys., 41, 782-811, 1990.

[10] M.C. Delfour and J.P. Zolésio, Shapes and Geometries: Analysis, Differential Calculus, and Optimization, SIAM, Philadelphia, 2001.

[11] Y. Dolak and C. Schmeiser, The Keller-Segel model with logistic sensitivity function and small diffusivity, SIAM J. Appl. Math., 66, 286-308, 2005.

[12] J. Escher, U.F. Mayer and G. Simonett, The surface diffusion flow for immersed hypersurfaces, SIAM J. Math. Anal., 29, 1419-1433, 1998.

[13] S. Evje, K.H. Karlsen and N.H. Risebro, A continuous dependence result for nonlinear degenerate parabolic equations with spatially dependent flux function, H. Freistühler and G. Warnecke, editors, Hyperbolic Problems: Theory, Numerics, Applications, Int. Series of Numerical Mathematics, Birkhäuser Verlag, 140, 1081-1104, 2001.

[14] J. Haskovec and C. Schmeiser, Transport in semiconductors at saturated velocities, Commun. in Math. Sci., 3, 219-233, 2005.

[15] T. Hillen and K. Painter, Global existence for a parabolic chemotaxis model with prevention of overcrowding, Adv. in Appl. Math., 26, 280-301, 2001.

[16] D. Horstmann, From 1970 until present: The Keller-Segel model in chemotaxis and its consequences, Part I, Jahresbericht der DMV, 105, 103-165, 2003.

[17] D. Horstmann, From 1970 until present: The Keller-Segel model in chemotaxis and its consequences, Part II, Jahresbericht der DMV, 106, 51-69, 2004.

[18] K.H. Karlsen and N.H. Risebro, On the uniqueness and stability of entropy solutions of nonlinear degenerate parabolic equations with rough coefficients, Discrete Contin. Dyn. Syst., 9, 1081-1104, 2003.

[19] E.F. Keller and L.A. Segel, Initiation of slime mold aggregation viewed as an instability, J. Theor. Biol., 26, 399-415, 1970.

[20] E.F. Keller and L.A. Segel, Model for chemotaxis, J. Theor. Biol., 30, 225-234, 1971.

[21] W.W. Mullins, Theory of thermal grooving, J. Appl. Phys., 28, 333-339, 1957.

[22] S.J. Osher and R.P. Fedkiw, The Level Set Method and Dynamic Implicit Surfaces, Springer, New York, 2002.

[23] S.J. Osher and J.A. Sethian, Fronts propagating with curvature-dependent speed: Algorithms based on Hamilton-Jacobi formulations, J. Comp. Phys., 79, 12-49, 1988. 
[24] K. Painter and T. Hillen, Volume-filling and quorum-sensing in models for chemosensitive movement, Canad. Appl. Math. Quart., 10, 280-301, 2003.

[25] C.S. Patlak, Random walk with persistence and external bias, Bull. Math. Biophys., 15, 311338, 1953.

[26] A.B. Potapov and T. Hillen, Metastability in chemotaxis models, J. Dyn. Diff. Eq., 17, 293-330, 2005.

[27] J. Simon, Compact sets in the space $L^{p}(0, T ; B)$, Anal. Math. Pura Appl., 146, 65-96, 1987.

[28] M.E. Taylor, Partial Differential Equations III, Springer, New York, 1996. 